\title{
New low-mass members of the Lupus 3 dark cloud: Further indications of pre-main-sequence evolution strongly affected by accretion $\star$
}

\author{
F. Comerón ${ }^{1}$, M. Fernández ${ }^{2}$, I. Baraffe ${ }^{3}$, R. Neuhäuser ${ }^{4,5}$, and A. A. Kaas ${ }^{6}$ \\ ${ }^{1}$ European Southern Observatory, Karl-Schwarzschild-Strasse 2, 85748 Garching, Germany \\ e-mail: fcomeron@eso.org \\ 2 Instituto de Astrofísica de Andalucía, CSIC, Apdo. 3004, 18080 Granada, Spain \\ e-mail: matilde@iaa.es \\ 3 C.R.A.L. (UMR 5574 CNRS), École Normale Supérieure, 69364 Lyon Cedex 07, France \\ e-mail: ibaraffe@ens-lyon. fr \\ ${ }^{4}$ Max-Planck-Institut für extraterrestrische Physik, Giessenbachstrasse 1, 85741 Garching bei München, Germany \\ 5 Astrophysikalisches Institut, Universität Jena, Schillergässchen 2-3, 07745 Jena, Germany \\ e-mail: rne@astro.uni-jena.de \\ ${ }^{6}$ Nordic Optical Telescope, Apartado 474, 38700 Santa Cruz de La Palma, Spain \\ e-mail: akaas@not.iac.es
}

Received 30 September 2002 / Accepted 28 May 2003

\begin{abstract}
A spectroscopic survey of a small area at the center of the Lupus 3 star-forming core has revealed four new mid-tolate M-type members, including a M7.5 brown dwarf. One of the new members, classified as M5, displays prominent forbidden lines and strong $\mathrm{H} \alpha$ emission $(E W(\mathrm{H} \alpha)=410 \AA)$, in addition to other permitted lines, and its luminosity is far below that of other members of the region with similar or later spectral types. We estimate a mass accretion rate rate of $\sim 1.4 \times 10^{-9} M_{\odot} \mathrm{yr}^{-1}$ for this object, although with uncertainties that do not exclude values as low as $10^{-10} M_{\odot} \mathrm{yr}^{-1}$. Based on the $\mathrm{H} \alpha /[\mathrm{SII}]$ ratio, the detection of $\mathrm{HeI}$, and the CaII infrared triplet, we argue that most of the $\mathrm{H} \alpha$ emission is produced near the surface of the object, probably in accretion columns or at the base of jets, rather than in a low-density extended region. The strong emission-line spectrum superimposed on an unusually faint photospheric continuum thus seems to be a real, intrinsic feature rather than a result of the viewing geometry caused by an edge-on disk blocking the light from the central object. Other Lupus 3 late-type members also display noticeable underluminosity, all of them having $E W(\mathrm{H} \alpha)>100 \AA$ as a result of the faint underlying continuum. We tentatively interpret these findings as evidence for the pre-main sequence evolution of objects with very low (possibly substellar) initial masses being significantly modified by accretion.
\end{abstract}

Key words. stars: pre-main sequence - stars: low-mass, brown dwarfs - stars: winds, outflows

\section{Introduction}

The Lupus star-forming complex is one of the belt of low mass star-forming regions within $\sim 200 \mathrm{pc}$ from the Sun, together with those in Chamaeleon, Rho Ophiuchi, R Coronae Australis, and Taurus. Physically, it belongs to the larger structure of the Scorpius-Centaurus OB association (Blaauw 1991), of which Lupus is currently one of the remaining active star forming sites together with the more conspicuous one of Rho Ophiuchi. The generic denomination of Lupus clouds applies to a number

Send offprint requests to: F. Comerón,

e-mail: fcomeron@eso.org

* Based on observations obtained at the European Southern Observatory using the Very Large Telescope in Cerro Paranal (program 67.C-0549(A)), the ESO New Technology Telescope in La Silla (program 69.B-0126(A)), and the 1.5 Danish telescope, also in La Silla (program 69.C-0423(B)). of distinct, loosely connected entities scattered over a large area of the sky and approximately at the same distance (e.g. Schwartz 1991). Its large-scale molecular contents as traced by ${ }^{12} \mathrm{CO}(J=1 \rightarrow 0)$ has been studied by Murphy et al. (1986), who estimated a total mass of $\sim 1.5 \times 10^{4} M_{\odot}$ from the integrated brightness temperature, well in agreement with the $1.7 \times 10^{4} M_{\odot}$ obtained in the more recent study of Tachihara et al. (2001) using the same tracer. Tachihara et al. (1996) studied individual molecular concentrations at a higher spatial resolution in ${ }^{13} \mathrm{CO}(J=1 \rightarrow 0)$, deriving individual masses in the range $100-1200 M_{\odot}$.

Comprehensive surveys of the pre-main sequence population associated to the Lupus clouds have been carried out by Schwartz (1977), who extended the list of $\mathrm{H} \alpha$-emitting members previously identified by Henize (1954) and Thé (1962), and by Wichmann et al. (1997) and Krautter et al. (1997). 
A detailed spectroscopic study of many of the members identified in Schwartz's work was presented by Hughes et al. (1994). Further studies on individual pre-main sequence members of the Lupus complex can be found in Reipurth \& Zinnecker (1993) and Nürnberger et al. (1997). Special attention has been paid to the Lupus 3 cloud, the one containing the richest stellar aggregate, where Nakajima et al. (2000) recently identified new faint, moderately obscured members with estimated masses near the substellar limit by means of nearinfrared imaging. The large-scale distribution of pre-main sequence stars in the Lupus clouds and their relationship to the concentrations of molecular gas has been studied by Tachihara et al. (2001).

Being one of the most nearby active star forming regions, the Lupus clouds are well suited for the detailed study of the individual properties of its pre-main sequence population. On the other hand, the Lupus clouds are in many respects similar, but not quite identical, to other nearby low-mass star forming regions that can be studied to the same level of depth and detail, thus allowing direct comparison between the stellar populations in those different aggregates and their relationship to the interstellar component out of which they are born (e.g. Chen et al. 1997). A particular aspect of interest in this context is the greater ratio of late M-type members with respect to earlier-type stars in the Lupus clouds, as compared to regions in principle similar like the Taurus clouds. This was already noted by Appenzeller et al. (1983) and confirmed by Hughes et al. (1994) and Wichmann et al. (1997). The new low mass members detected by Nakajima et al. (2000) are consistent with this feature of the Lupus population.

In this paper we present the results of a survey of a small area at the core of the Lupus 3 cloud, as signaled by the Herbig Ae star HR 5999 and its optical companion HR 6000, in which we have identified new low mass members by means of their characteristic $\mathrm{H} \alpha$ emission and have obtained good quality spectra of previously known low-mass members. The $\mathrm{H} \alpha$ equivalent widths measured for the members of our sample span a wide range of values, making them suitable for addressing the possible effects of accretion activity, as traced by $\mathrm{H} \alpha$ emission, on the actual tracks followed by pre-main sequence objects in the temperature-luminosity diagram. Strong accretion has been recently suggested to dramatically shift the position of very low mass stars and brown dwarfs in that diagram (Fernández \& Comerón 2001), and new supporting observational evidence is discussed in this paper.

\section{Observations}

\subsection{Spectroscopy}

The spectroscopic observations presented in this paper were obtained using the FORS1 visible-light camera and spectrograph at Antu, Unit Telescope 1 of the ESO Very Large Telescope. Short images (30 s) of overlapping fields around the HR 5999/HR 6000 pair through the Bessell I filter were obtained on the night of 29/30 April 2001 in order to provide a reference image for the slitless spectra extraction. Slitless spectroscopy was then obtained on the night of 1/2 May 2001 of the two fields immediately East and West of HR 5999/HR 6000, using an instrumental configuration consisting of the $300 \mathrm{~V}$ grism used simultaneously with the Gunn $r$ filter, to isolate the region around $\mathrm{H} \alpha$ between $6120 \AA$ and $6940 \AA$. The resolution yielded by this instrumental setup, in the prevailing seeing conditions of $0^{\prime \prime} 8$ under which our observations were taken, is approximately $11 \AA$ per resolution element. Each of the two fields was imaged for one hour, split in three exposures with small telescope offsets perpendicular to the dispersion direction in between.

The slitless spectroscopy images were reduced and combined in near-real time by means of dedicated IRAF scripts that automatically detected stars in the images and extracted their 1D spectra from the slitless spectroscopy image (see Comerón et al. 2000 for a detailed description of this procedure). Emission-line stars with late-type underlying continua were then selected by visual inspection of the extracted spectra for follow-up multi-object spectroscopy (MOS).

The movable-blades facility available in FORS 1 allowed us to fully define the instrument setup for the ensuing MOS, including the masks to be used, while the second field was being observed in slitless mode. Thanks to this, the MOS observations were performed immediately after the slitless ones on the same night of 1/2 May 2001. The setup used consisted of the 300I grism yielding a resolution similar to that of the slitless spectra, but now covering a more extended range between approximately $6000 \AA$ and $11000 \AA$. The actual range for each object depended on its position in the field. Three exposures were taken through each mask, each of $10 \mathrm{~min}$ in duration, at telescope positions slightly offset along the slits.

\subsection{Imaging}

Besides the images obtained as a reference for the slitless spectroscopy with the VLT, we obtained $V R_{\mathrm{C}} i$ images with DFOSC at the $1.5 \mathrm{~m}$ Danish telescope in La Silla on the nights of 22/23 and 23/24 March 2002 to determine the broad-band spectral energy distribution of the newly found members. Three dithered images were obtained of each of the two overlapping fields East and West of HR 5999/HR 6000. Thanks to the larger field of view of DFOSC, these images included the fields for which slitless spectroscopy had been obtained the previous year as well as some of the surrounding area. The combined images add up to effective exposure times of $120 \mathrm{~s}$ in $V, 60 \mathrm{~s}$ in $R_{\mathrm{C}}$, and $45 \mathrm{~s}$ in $i$. Photometry of the stars observed in MOS was performed on the DFOSC images using the PHOT task under the APPHOT package in IRAF. Given the uncrowdedness of the field and the lack of prominent nebulosity, the capabilities of PHOT were deemed sufficient for our purposes. For the sake of comparison with other studies, we have transformed the $i$ magnitudes to $I_{\mathrm{C}}$ magnitudes using Eqs. (23) of Fukugita et al. (1996).

The broad-band photometry in the visible was complemented with near-infrared $J H K_{\mathrm{s}}$ imaging obtained with SOFI at the New Technology Telescope (NTT) on 19 August 2001 and 20 July 2002. The August 2001 data contain $J$-band imaging for all the objects, while the July 2002 ones 
provide $J H K_{\mathrm{s}}$ imaging for the central parts of the FORS1 field. In both cases, dithered images of the fields East and West of HR 5999/HR 6000 were obtained, sky-subtracted by median filtering of the individual frames without correcting for the telescope offsets, and combined by shift-and-add. The photometry on the reduced frames was performed using also PHOT, like in the previously described set. A caveat to keep in mind is that the visible and infrared observations are non-simultaneous. Therefore, the possibility of mismatches between the visible and the near-infrared observations due to time variability, which is discussed in Sect. 3.1, cannot be excluded.

\subsection{The distance to Lupus 3}

Before entering in the description and discussion of our results, it seems convenient to consider in some detail the distance to the Lupus 3 star forming region, as this is a necessary ingredient (and an important caveat) in the estimate of luminosities that we discuss below. Most studies have determined or adopted distances in the range 130-170 pc on the basis of the membership in the Scorpius-Centaurus association (see e.g. the discussion in Murphy et al. 1986, and also Krautter 1991) or by estimating spectroscopic parallaxes for early-type surrounding stars that are expected to belong also to Scopius-Centaurus (Hughes et al. 1993). A distance as short as $100 \mathrm{pc}$ has been suggested by Knude \& Høg (1998) based on the extinction distribution of foreground and background stars with distances measured by Hipparcos. The best value currently available for the distance to the Upper Centaurus-Lupus subgroup of the Scorpius-Centaurus association is $140 \pm 2 \mathrm{pc}$, derived by de Zeeuw et al. (1999) based on Hipparcos trigonometric parallaxes. Murphy et al. (1986) have suggested that the Lupus complex, while still physically belonging to Scorpius-Centaurus, may be in front of it, with the extinction produced by the cloud being responsible for the lower number of $\mathrm{OB}$ stars in that direction.

The trigonometric parallaxes measured by Hipparcos for HR 5999 and HR 6000 allow in principle a more direct derivation of the distance to Lupus 3. Somewhat surprisingly, the derived distances for these stars are $207 \mathrm{pc}$ and $241 \mathrm{pc}$ respectively, the uncertainty being in both cases approximately $\pm 40 \mathrm{pc}$. This determination is not incompatible with the normally adopted distance to the bulk of Scorpius-Centaurus, which is considerably extended in the plane of the sky: assuming that the depth is of the same order as its spread in the sky, a distance of $\sim 200 \mathrm{pc}$ of Lupus 3 places it in the far side of the association rather than outside it. We note that a similarly high distance of $190 \pm 27 \mathrm{pc}$ to the Lupus star forming complex, based on a larger set of Hipparcos distances, was derived by Wichmann et al. (1998).

To be consistent with the individual distances to HR 5999 and HR 6000, with that derived by Wichmann et al. (1998), and with the membership in Scorpius-Centaurus, we have opted for the rather eclectic solution of adopting a distance of $200 \mathrm{pc}$ for Lupus 3, corresponding to a distance modulus of $6.5 \mathrm{mag}$. We should stress the important difference that this implies with respect to distances adopted in previous works: by comparison to Hughes et al. (1994), our distance modulus is $0.8 \mathrm{mag}$ greater than in that work, and it would reach one full magnitude if we adopted the sheer average of the Hipparcos trigonometric parallaxes for HR 5999 and HR 6000. Luminosities may thus change by a factor of two depending on which distance modulus (the one of Hughes et al. or the one adopted in this work) is assumed. It is important to bear in mind that the distance uncertainty is by far the dominating factor of uncertainty in the luminosities derived for individual members of Lupus 3. However, we do not consider that depth effects contribute to relative differences in luminosity among the members. Judging from the extent of the Lupus 3 cloud on the sky from Digitized Sky Survey images, we estimate its physical size to be between 4 and $5 \mathrm{pc}$ along the largest dimension projected on the sky. A size at least twice as large along the line of sight would be required to produce differences in luminosity exceeding the $10 \%$ level. We thus consider it highly unlikely that depth yields any significant contribution to the spread in luminosities among the objects discussed here.

\section{Results}

The review of the spectra obtained in our slitless images revealed all the previously known $\mathrm{T}$ Tauri stars in the observed region plus four additional, previously unrecognized emissionline objects. Figure 1 shows the composite of two FORS1 $I$-band images of the surveyed field indicating the positions of all the spectroscopically identified members of the star forming region, either from Hughes et al. (1994) or from the present work. It should be noted that a few additional possible faint members, not plotted there, have been reported by Nakajima et al. (2000) based on their infrared colors. Given the constraints due to the number of slits that can be defined in a single setting of FORS1 and their relative positions, it was not possible to obtain MOS spectra of all $\mathrm{H} \alpha$-emitting stars in the region, both new and previously known. Instead, our selection for MOS targets focused on the newly found $\mathrm{H} \alpha$-emitting objects, and previously known members were observed only if it was feasible to position a slit on them with the same setting. Preference was given however to faint objects suspected to have late-type slitless spectra but no discernible $\mathrm{H} \alpha$ emission rather than to bright known members. However, none of those faint objects was confirmed as a candidate Lupus member. Other than the object labelled Par-Lup3-4, none of the candidate faint members in Nakajima et al. (2000) survey was included in our spectroscopic sample.

The positional and photometric data of the objects for which MOS spectra were obtained are given in Table 1 together with their spectral type (see Sect. 3.2). The nomenclature for all previously known members refers to Schwartz's (1977) catalog, although some of them have other denominations as well; we note in particular Sz102 = Th 28 (Thé 1962), also known as Krautter's star. For the new members, we have followed the same criterion as in Fernández \& Comerón (2001) and thus denominate them generically as Par-Lup3, where "Par" stands for Paranal. As it turned out, the MOS setting serendipitously included a fifth late-type object near the position of one of the four new $\mathrm{H} \alpha$-emitting objects, which we have denominated 


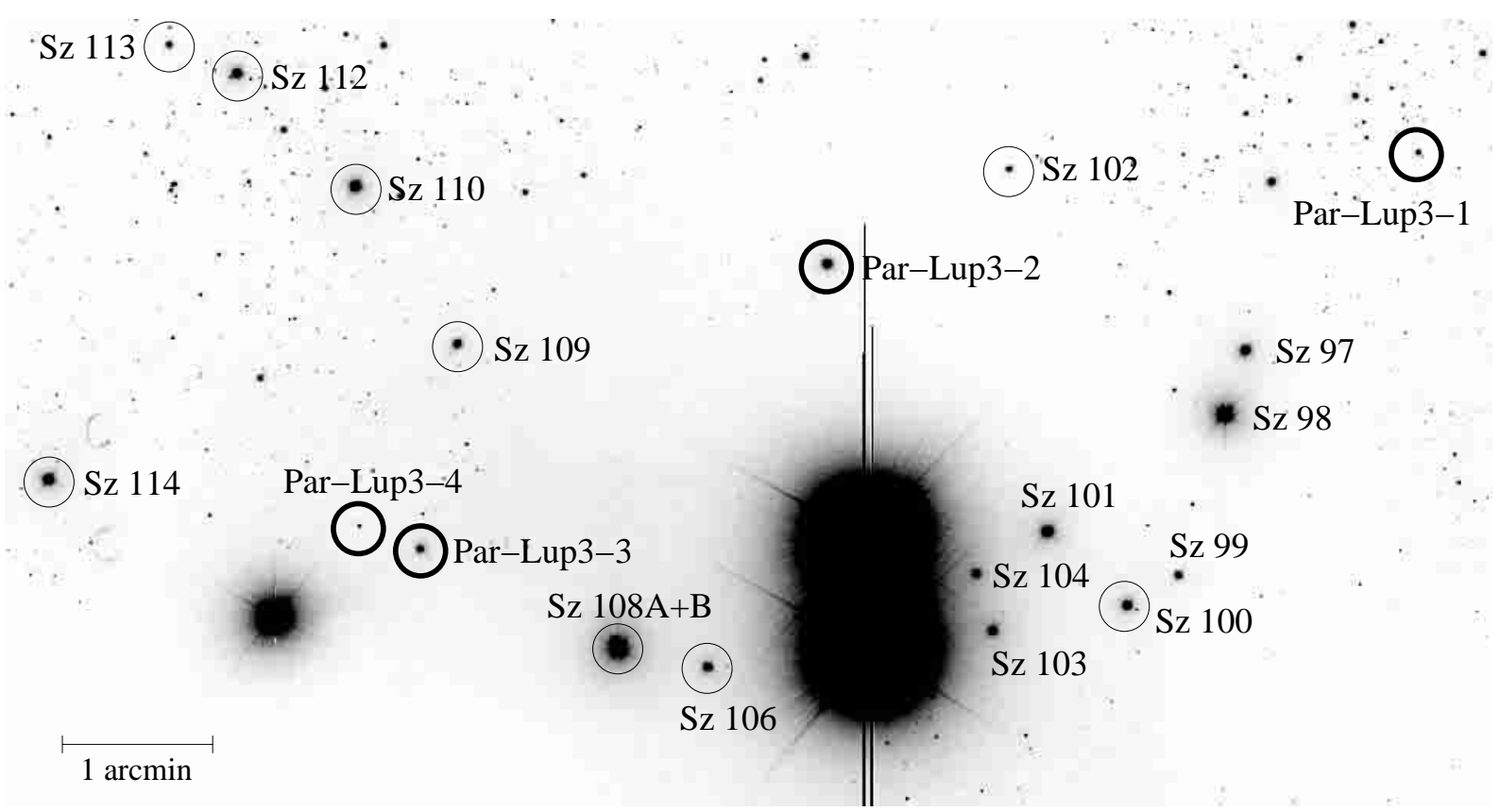

Fig. 1. A composite of two FORS1 I-band images indicating the positions of previously and newly identified objects. The field measures $9.67^{\prime} \times 5^{\prime} 13$, with North to the top and East to the left. The two bright stars below the center are HR 5999 (bottom) and HR 6000 (top). Bold circles indicate new members identified in this work, and light circles are previously known members for which new spectra are presented here. Other known members for which no new spectroscopy has been obtained are also indicated.

Table 1. Positions, photometry, and spectral types of the observed stars.

\begin{tabular}{lcccccccc}
\hline \hline Object & IAU designation & $V$ & $R$ & $I_{\mathrm{C}}$ & $J$ & $H$ & $K_{\mathrm{s}}$ & sp. type \\
\hline Sz 100 & J160825.8-390601 & 16.53 & 14.95 & 13.09 & 11.02 & 10.36 & 9.84 & M5 \\
Sz 102 & J160829.7-390311 & 16.93 & 15.60 & 15.08 & $14.41^{1}$ & $13.67^{1}$ & $12.51^{1}$ & $?$ \\
Sz 106 & J160839.7-390624 & & & & 12.92 & 11.82 & 10.86 & M2.5 \\
Sz 108A & J160842.7-390617 & & & & $9.76^{1}$ & $9.09^{1}$ & $8.78^{1}$ & M3 \\
Sz 108B & J160842.8-390614 & & & & 12.04 & 11.32 & 10.82 & M6 \\
Sz 109 & J160848.1-390418 & 17.43 & 16.03 & 13.87 & 11.40 & 10.92 & 10.55 & M6.5 \\
Sz 110 & J160851.5-390317 & 14.69 & 13.77 & 12.42 & $11.22^{1}$ & $10.27^{1}$ & $9.83^{1}$ & M4.5 \\
Sz 112 & J160855.4-390233 & 16.37 & 15.05 & 13.13 & $11.05^{1}$ & $10.34^{1}$ & $9.85^{1}$ & M6 \\
Sz 113 & J160857.7-390222 & 18.16 & 16.75 & 14.85 & $12.86^{1}$ & $12.08^{1}$ & $11.58^{1}$ & M6 \\
Sz 114 & J160901.7-390511 & 15.47 & 14.23 & 12.49 & $10.42^{1}$ & $9.62^{1}$ & $9.18^{1}$ & M5.5 \\
Par-Lup3-1 & J160815.9-390307 & 19.92 & 17.60 & 15.45 & $12.60^{2}$ & & & M7.5 \\
Par-Lup3-1/cc 1 & J160816.0-390305 & & & $20.5^{3}$ & & & & M5: \\
Par-Lup3-2 & J160835.7-390348 & 16.76 & 15.18 & 13.21 & 11.48 & 10.97 & 10.87 & M6 \\
Par-Lup3-3 & J160849.3-390538 & 17.71 & 16.43 & 14.41 & 11.43 & 10.26 & 9.63 & M4.5 \\
Par-Lup3-4 & J160851.3-390529 & $21.9:$ & 19.78 & 18.33 & 16.42 & 15.09 & 13.82 & M5 \\
\hline $\begin{array}{l}\text { Notes: } \\
\text { 1 JHK magnitudes from Hughes et al. (1994). }\end{array}$ & & & & & & \\
2 J magnitude from the June 2001 observations. & & & & & & \\
3 The value given is actually the $I_{c}$ magnitude from the FORS1 image. & & &
\end{tabular}

Par-Lup3-1/cc 1 following the suggestion of Neuhäuser et al. (2002) on naming of companion candidates. Its actual relationship to Lupus 3 is very doubtful, as discussed below. Unless otherwise indicated, the $V R_{\mathrm{C}} I_{\mathrm{C}}$ photometry is from our DFOSC observations and the $J H K$ photometry from our July $2002 \mathrm{ob}-$ servations. Unfortunately, several of the Sz stars observed with FORS1 in MOS as well as Par-Lup3-1 were out of the field of view in the July 2002. In those cases, the $J H K$ photometry has been adopted from Hughes et al. (1994) or from the $J$-band August 2001 observations for Par-Lup3-4.

\subsection{Photometry and variability}

It is interesting to compare our photometry with that published by Hughes et al. (1994) in the visible and the infrared and with that of Nakajima et al. (2000) in the infrared, as well as the 
two epochs in $J$ available for some of the objects from our own observations. Most objects display at most the moderate variability characteristic of T Tauri stars with amplitudes typically $<0.3 \mathrm{mag}$ in all the bands. This is the case of Sz 100, Sz 106, $\mathrm{Sz} 108 \mathrm{~B}, \mathrm{Sz} 110, \mathrm{Sz} 112$, and Sz 114. A comparison between the June 2001 and July $2002 \mathrm{~J}$-band photometry also shows differences within this range for Par-Lup3-2 and Par-Lup3-3, while only one-epoch data are available for Par-Lup3-1, as well as for Sz 108A due either to scattered light from HR 5999/6000 or to saturation in the infrared. Greater differences are seen however for other objects indicating large-amplitude variability. For $\mathrm{Sz} 102$ we obtain $V$ and $I_{\mathrm{C}}$ magnitudes that are fainter than those in Hughes et al. (1994) by 0.51 and 0.25 mag respectively, the $R_{\mathrm{C}}$ magnitude staying approximately equal. Sz 109 appears distinctly redder in our DFOSC observations than in the data of Hughes et al. (1994): while our $I_{\mathrm{C}}$ magnitude is only 0.05 mag fainter than in that work, $V$ and $R_{\mathrm{C}}$ are fainter by $0.91 \mathrm{mag}$ and $0.65 \mathrm{mag}$, respectively. The large variability of this object has been also noted by Nakajima et al. (2000), who found it to be at least 0.2 mag brighter in $J$ than Hughes et al. (1994). Also Sz 114 is fainter and redder in our observations than in those of Hughes et al. (1994) by $1.04 \mathrm{mag}(V)$, $0.66 \mathrm{mag}\left(R_{\mathrm{C}}\right)$ and $0.34 \mathrm{mag}\left(I_{\mathrm{C}}\right)$. The features in the $V R I$ variability may be explained in principle by the appearance and disappearance (due to rotation or spot lifetime) of surface regions at a temperature different from the normal photosphere, possibly hot regions at the bottom of the accretion columns. Further photometric monitoring is needed to confirm or disprove this interpretation.

In the infrared, differences above the 0.3 mag level are found only for Par-Lup3-4 by comparing our July $2002 \mathrm{JHK}_{\mathrm{S}}$ photometry with that of Nakajima et al. (2000). This object was clearly fainter and redder in July 2002, with a difference with respect to Nakajima's data of $0.71 \mathrm{mag}(J), 0.37 \mathrm{mag}(H)$ and $0.12 \mathrm{mag}\left(K_{\mathrm{s}}\right)$. The $J$ magnitude observed in June 2001 is also brighter than in July 2002 by 0.45 mag. These changes in the brightness and colors are one of the peculiar features of Par-Lup3-4, together with others that will be discussed in this paper.

\subsection{Spectral classification}

We find a clear systematic difference between our assigned spectral types and those of Hughes et al. (1994), in the sense of ours being $\sim 2$ spectral subclasses later (we exclude from this comparison Sz102, whose spectrum is featureless in the considered range except for the abundant emission lines and the telluric absorption bands, thus precluding a spectral classification). Since the spectra themselves are not included in Hughes et al.'s paper, we cannot reassess their classification to attempt to identify the origin of the discrepancy. However, we suspect that the reason may be related to the spectral ranges used in their work and ours. Our classification is based on a comparison to the spectral sequence for K-to-late M stars of Kirkpatrick et al. (1991) and, given the quality of our spectra, we estimate it to be accurate to within half a spectral subclass. The comparison makes use of temperature-sensitive features like the shape of the broad continuum peak between $\sim 7300 \AA$ and $\sim 7600 \AA$, the change of slope that occurs at $\sim 8000 \AA$, and the height of the rise between $\sim 8000 \AA$ and $\sim 8150 \AA$. For late $\mathrm{M}$ spectra these features provide a sensitive sequence allowing the accurate determination of spectral subtypes down to at least M8, also for very young objects (see e.g. Fig. 2 of Comerón et al. 2000). On their side, Hughes et al. (1994) base their classification on the 5800-7000 $\AA$ interval, which is probably better suited for the classification of earlier spectra. It is also somewhat more prone to veiling as the continuum emission due to accretion increases in importance towards the blue with respect to the underlying photospheric continuum, which might explain at least in part why the classification based in that bluer range is systematically earlier. Since the 6500-9000 А range has been used in most recent discussions on the classification of late M- and L-type objects in star forming regions, and for the sake of consistency with previous work by us and other authors, we consider it more appropriate for the current study. Analogous reasons of self-consistency in the analysis of the data have led us not to consider the other members in the field that were not observed and classified by us.

We have checked our spectral classification against qualitative criteria based on indices that measure the strength of temperature-sensitive features. The $R_{4} / R_{7}$ index proposed by Prosser et al. (1991), which measures the height of the local peak between $7000 \AA$ and $7068 \AA$ ( $R_{7}$ passband) with respect to the nearby depressed continuum between $6750 \AA$ and $6844 \AA$ ( $R_{4}$ passband), yields an appropriate determination of the spectral type over the whole late-K to mid-M range and can be applied also at later $\mathrm{M}$ types, as described by Comerón et al. (2000). For types later than about M6.5, the VO index defined by Kirkpatrick et al. (1995) measuring the depth of the VO feature near $7450 \AA$ provides also a sensitive spectral type indicator. Overall, we find an excellent agreement to within half a spectral subclass for all the objects that we classify as M6 or later. At earlier types, for which only the $R_{4} / R_{7}$ index is sensitive, we find a precise agreement for Par-Lup3-4 at spectral type M5. For the other objects, our assigned spectral types are between half and one spectral subclass earlier than those derived from the $R_{4} / R_{7}$ vs. spectral type calibration of Prosser et al. (1991). We suspect that the offset may be due to systematic differences between the spectra of the young objects that we are considering here and the evolved field stars that were used in that paper to calibrate the relationship between that index and the spectral type. It is important to note that the $R_{4} / R_{7}$ index is defined near the blue edge of the spectral range that we use for classification, and may thus be expected to be more sensitive to veiling. The effect of veiling should be an increase in the value of the index due to the dilution of the peak sampled by $R_{7}$ and resulting in the assignation of a spectral type earlier than the unveiled one. This effect goes into the direction opposite to the systematic offset that we find in our data, which leads us to conclude that veiling may introduce at most a small bias in our spectral type determination, most probably not exceeding one spectral subclass.

Several features of the very low mass members of this region of Lupus 3 are already visible at a glance in Fig. 2. M types later than M5 are abundant, also among objects previously 


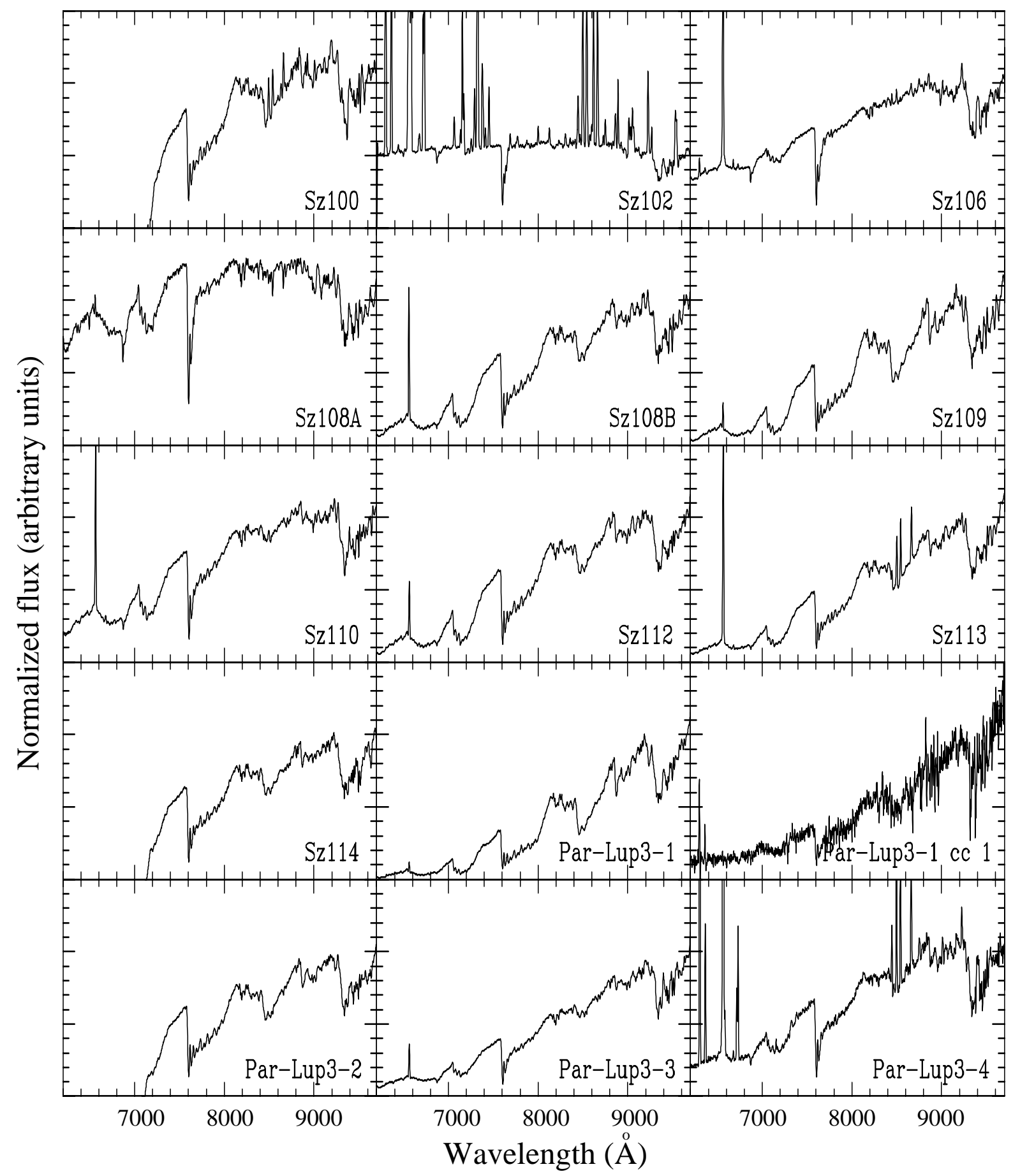

Fig. 2. All the FORS1 spectra obtained in the observations described in Sect. 2.1. To homogenize the presentation, all the spectra have been normalized to the flux in the 9000-10000 $\AA$ interval. The vertical scale has been further reduced by a factor of 2 in the case of Sz 102 .

classified by Hughes et al. (1994), and one of the new objects is classified as late as M7.5. Secondly, the $\mathrm{H} \alpha$ equivalent widths have large variations as observed in other star forming regions, but reach rather extreme values with respect to the underlying continuum in the well-known case of Sz102 and the newly found Par-Lup3-4. The emission spectrum of these objects is discussed in further detail below.

The classification is rather uncertain for Par-Lup3-1/cc 1, the faint object $3^{\prime \prime}$ southwest of the M7.5 object Par-Lup3-1. The fact that its spectrum is clearly that of a late-type star, that it is so close along the line of sight to the latest-type $\mathrm{H} \alpha$-emitting member identified in the star forming region, and that it is much fainter than it, led us to consider the possibility that Par-Lup3-1/cc 1 might be a bound substellar companion to Par-Lup3-1 with a spectral type later than M. However, a comparison to the sequence of late $\mathrm{M}$ and $\mathrm{L}$ spectral types published by Kirkpatrick et al. (1999) shows that the features that can be discerned in the spectrum of Par-Lup3-1/cc 1 are clearly M-like and exclude the possibility of a very late $\mathrm{M}$ or $\mathrm{L}$ type. We rather consider Par-Lup3-1/cc 1 a likely distant mid-M giant, 
Table 2. Equivalent widths of the main emission lines observed in the spectra of the stars discussed in this paper.

\begin{tabular}{|c|c|c|c|c|c|c|c|c|c|c|c|c|c|}
\hline Object & $\begin{array}{l}{[\mathrm{OI}]} \\
6300\end{array}$ & $\begin{array}{l}{[\mathrm{OI}]} \\
6364\end{array}$ & $\begin{array}{c}\mathrm{H} \alpha \\
6563\end{array}$ & $\begin{array}{l}{[\mathrm{NII}]} \\
6583\end{array}$ & $\begin{array}{c}\mathrm{HeI} \\
6678\end{array}$ & $\begin{array}{l}{[\mathrm{SII}]} \\
6716\end{array}$ & $\begin{array}{l}{[\mathrm{SII}]} \\
6731\end{array}$ & $\begin{array}{l}{[\mathrm{OII}]} \\
7319\end{array}$ & $\begin{array}{l}{[\mathrm{OII}]} \\
7329\end{array}$ & $\begin{array}{c}\text { OI } \\
8446\end{array}$ & $\begin{array}{l}\text { CaII } \\
8498\end{array}$ & $\begin{array}{l}\text { CaII } \\
8542\end{array}$ & $\begin{array}{r}\text { CaII } \\
8662\end{array}$ \\
\hline Sz100 & \multicolumn{7}{|c|}{ (84) } & & - & - & 5.4 & 5.0 & 4.3 \\
\hline Sz102 & 157 & 50.5 & 445 & 44 & 4: & 19 & 43 & \multicolumn{2}{|c|}{70.4} & 7.3 & 24.9 & 31 & 25 \\
\hline Sz106 & 3.3 & 0.7 & \multirow{2}{*}{\multicolumn{2}{|c|}{110}} & 1.5 & \multicolumn{2}{|c|}{0.9} & - & - & 0.8 & 1.0 & 0.2 : & 0.6 : \\
\hline Sz108A & & & & & & - & - & - & - & - & - & - & - \\
\hline Sz108B & 1.6 & - & \multicolumn{2}{|c|}{53} & - & - & - & - & - & - & - & - & - \\
\hline Sz109 & - & - & \multicolumn{2}{|c|}{15} & - & - & - & - & - & - & - & - & - \\
\hline Sz110 & - & - & \multicolumn{2}{|c|}{42} & 2.2 & - & - & - & - & - & - & - & - \\
\hline Sz112 & - & - & \multicolumn{2}{|c|}{23.5} & & - & - & - & - & - & - & - & - \\
\hline Sz113 & - & - & \multirow{2}{*}{\multicolumn{2}{|c|}{$\begin{array}{l}139 \\
(24)\end{array}$}} & 2.6 & - & - & - & - & - & 8.0 & 8.0 & 6.0 \\
\hline Sz114 & & & & & & & & - & - & - & - & - & \\
\hline Par-Lup3-1 & - & - & \multirow{2}{*}{\multicolumn{2}{|c|}{$\begin{array}{c}12 \\
(6.5)\end{array}$}} & - & - & - & - & - & - & - & - & - \\
\hline Par-Lup3-2 & & & & & & & & - & - & - & - & - & \\
\hline Par-Lup3-3 & & & \multicolumn{2}{|c|}{19} & - & - & - & - & - & - & - & - & - \\
\hline Par-Lup3-4 & 133 & 42 & 410 & 9: & 1.6 & 21 & 38 & \multicolumn{2}{|c|}{ 3: } & 5.5 & 14.5 & 13.8 & 10.0 \\
\hline LS-RCrA 1 & 92 & 25 & 360 & 15 & 1.5 & 12.4 & 19.2 & 3.6 & 3.6 & & 4.0 & 3.6 & 2.2 \\
\hline
\end{tabular}

\section{Notes:}

$\mathrm{H} \alpha$ in parentheses are those measured on the slitless spectra.

The symbol "_-" indicates that the line is not detectable, while a blank entry means that the line falls outside the spectral range covered for that object.

a possibility that appears reasonable in view of the relatively low galactic latitude of Lupus 3 and the fact that Par-Lup3-1 is projected on an area of low dust column density, as is obvious from the high stellar density in this direction. Therefore, we will not consider Par-Lup3-1/cc 1 among the members of Lupus 3 in the rest of this paper.

\subsection{Emission-line spectra}

Perhaps the most striking feature of the sample of objects considered in this paper is the variety of emission line spectra found and the existence of some rather extreme cases worth considering in some detail.

Table 2 lists the equivalent width of the main lines and, for comparison, the same information for LS-RCrA 1 (Fernández $\&$ Comerón 2001). The similarity of one of the newly found objects, Par-Lup3-4, with LS-RCrA 1 is remarkable as illustrated by Fig. 3. The emission lines have their origins in the different components associated to the accretion/mass loss processes that accompany the early stages of stellar evolution and that have been extensively studied in stars of somewhat higher masses than those considered here (e.g. Hartigan et al. 1995; Hirth et al. 1997). Forbidden lines such as those seen in the spectra of Sz102, Sz106, and Par-Lup3-4, as well as some amount of $\mathrm{H} \alpha$ emission, are commonly observed in actively accreting young stars. These lines are thought to have their origin in a magnetically driven wind stemming from regions in the vicinity of the star (typically $<1 \mathrm{AU}$ ), either in the star/disk magnetopause (Shang et al. 2002; see also Kwan \& Tademaru 1988; Hirth et al. 1997) or a more extended range in radii (Cabrit et al. 1999). The forbidden-line emission spectrum is similar to those of the closely related Herbig-Haro objects.

In the commonly accepted magnetospheric accretion model, the circumstellar disk is magnetically truncated near the surface of the star (Königl 1991), with material flowing from the disk towards the star in accretion columns that follow the magnetic field lines connecting the inner edge of the disk with high latitude regions of the star. Much of the permittedline emission, particularly $\mathrm{H} \alpha$, is thought to have its origin in these columns, as confirmed by the broad velocity profiles observed in $\mathrm{H} \alpha$ (Muzerolle et al. 1998a, 2000). At the base of these accretion columns, close to the surface of the star, shock fronts develop creating a high temperature layer (Königl 1991; Gullbring 1994; Lamzin 1995). Much of the HeI emission, the broad component of the CaII infrared triplet lines (and possibly also the narrow component according to Batalha et al. 1996), and the continuum emission at short wavelengths veiling the photospheric spectrum, are thought to arise in the hot spots at the base of the accretion columns. An additional contribution to the $\mathrm{H} \alpha$ emission in the vicinity of the star may arise from the base of a collimated jet, also near its surface (Takami et al. 2001, 2003).

Many of the detailed diagnostics on the physical conditions and the structure of the regions where both the outflow and accretion signatures originate can be derived from high resolution spectra, as yet unavailable for our sources. Nevertheless, the lines visible in our low-resolution spectra give already some very useful directions. The wide range of $\mathrm{H} \alpha$ intensities found in our sample, spanning over two orders of magnitude, hints to a range of accretion activities. On the other hand, also forbidden lines associated to outflows display intensities ranging from undetectable to the extreme case of Sz102, with varying levels of correlation to the lines associated to accretion.

\subsubsection{Accretion signatures}

Most of the CaII infrared triplet emission, if not all of it, is likely to be due to the mass accretion process. Although 

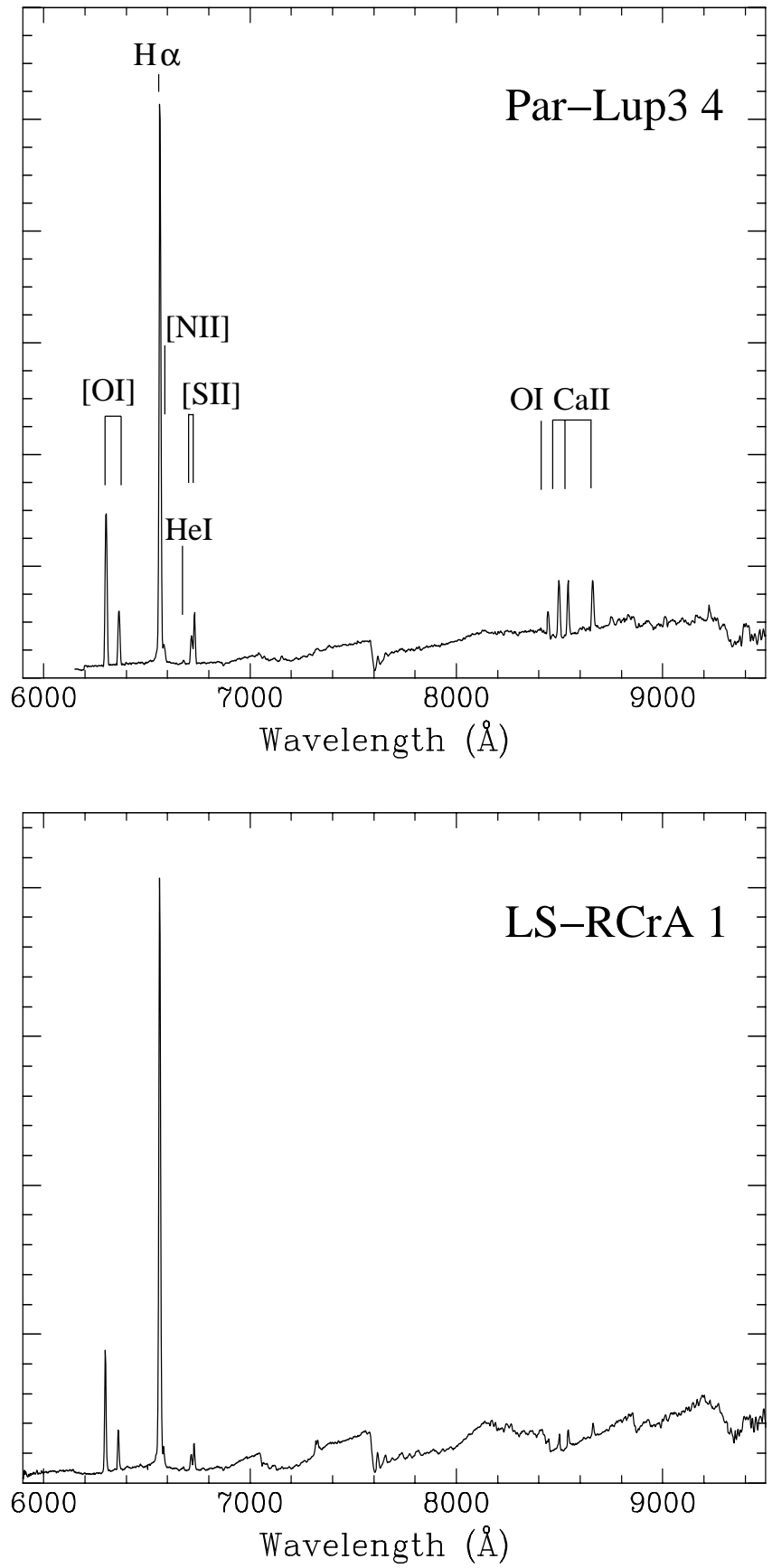

Fig. 3. Spectrum of Par-Lup3-4 with the main emission lines identified. For comparison, the spectrum of LS-RCrA 1 (from Fernández \& Comerón 2001) is shown.

the structure of the lines is unresolved in our spectra, the equivalent widths of the CaII triplet at $8498 \AA, 8542 \AA$, and $8662 \AA$ that we detect in Sz100, Sz102, Sz113, Par-Lup3-4, and LS-RCrA 1, are well above those typically resulting from chromospheric activity in young late-type stars as well as those of the narrow-band component possibly related to accretion (Batalha et al. 1996). However, they are within the range observed in the broad-line component (Hamann \& Persson 1992) that is generally attributed to accretion. This is therefore the most likely origin also in our objects. In all cases the line ratios are much closer to $1: 1: 1$ than to the expected ratio 1:9:5
Table 3. Estimated accretion rates from the CaII(18542) line.

\begin{tabular}{lcc}
\hline \hline Object & $\mathcal{F}_{\text {CaII }(28542)}\left(\mathrm{erg} \mathrm{s}^{-1}\right)$ & $\dot{M}\left(M_{\odot} \mathrm{yr}^{-1}\right)$ \\
\hline Sz100 & $5.2 \times 10^{29}$ & $1.9 \times 10^{-8}$ \\
Sz102 & $2.0 \times 10^{29}$ & $7.9 \times 10^{-9}$ \\
Sz106 & $2.0 \times 10^{28}$ & $1.0 \times 10^{-9}$ \\
Sz113 & $1.8 \times 10^{29}$ & $7.2 \times 10^{-9}$ \\
Par-Lup3-4 & $2.8 \times 10^{28}$ & $1.4 \times 10^{-9}$ \\
LS-RCrA 1 & $4.7 \times 10^{27}$ & $2.8 \times 10^{-10}$ \\
\hline
\end{tabular}

${ }^{1} I_{\mathrm{C}}$ estimated from the FORS1 images.

for optically thin emission, as noted by Fernández \& Comerón (2001) for the case of LS-RCrA 1 and as usually found among T Tauri stars (Herbig \& Soderblom 1980) and sources powering Herbig-Haro outflows (Reipurth et al. 1986). The absence of any detectable forbidden [CaII] emission at 7291 and $7324 \AA$ suggests indeed that the observed CaII emission arises in a high density region $\left(N_{\mathrm{e}}>10^{-7} \mathrm{~cm}^{-3}\right)$, under conditions found near the surface of the object, rather than in a lower density outflow. As argued below, the weakness of the forbidden lines in Sz 106 suggests that the contribution of the outflow emission to the $\mathrm{H} \alpha$ flux is small. Otherwise, the CaII infrared triplet is poorly correlated with $\mathrm{H} \alpha$ among our objects.

Muzerolle et al. (1998b) have examined the correlation between the accretion rates as derived from ultraviolet veiling and the intensity of different emission lines, finding that the tightest match occurs for the CaII infrared triplet. For the correlation

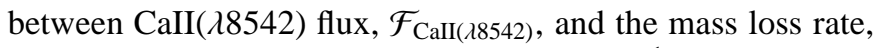
the linear fit to their Fig. 9 can be expressed as ${ }^{1}$

$\log \dot{M}\left(M_{\odot} \mathrm{yr}^{-1}\right)=-34.15+0.89 \log \mathcal{F}_{\mathrm{CaII}(\lambda 8542)}\left(\mathrm{erg} \mathrm{s}^{-1}\right)$.

We derive the flux in the line as

$\mathcal{F}\left(\mathrm{erg} \mathrm{s}^{-1}\right)=4.72 \times 10^{33} E W(\AA) \times 10^{-0.4\left(m_{\lambda}-0.54 A_{V}\right)}$

where $m_{\lambda}$ is the magnitude of the star at the wavelength of the line, calculated from the flux in the spectrum relative to the $I_{\mathrm{C}}$ band and scaling it to the absolute flux in $I_{\mathrm{C}}$, using the zero magnitude fluxes of Bessell (1979). $A_{V}$ is the visual extinction, to be discussed in Sect. 3.4, and the coefficient 0.54 to convert it to the extinction at $8542 \AA$ is obtained by interpolating the extinction law of Mathis (1990). Applying (1) and (2) to all the stars with detectable CaII emission, we obtain the mass loss rates given in Table 3 .

The HeI line, weakly appearing in the spectra of Sz102, Sz106, Sz110, Sz113, and Par-Lup3-4, as well as LS-RCrA 1, may be formed in principle either in the accretion shock (Lamzin et al. 1996) or in the disk wind, provided that sufficiently high temperatures are produced (Beristain et al. 2001).

\footnotetext{
${ }^{1}$ Note that Muzerolle et al. (1998b) use in their plots the flux emitted per unit surface, adopting a fiducial radius of $2 R_{\odot}$ for classical $\mathrm{T}$ Tauri stars. We rather use the flux integrated over the entire surface in Eq. (1), assuming that the correlation found by Muzerolle et al., which spans over three orders of magnitude in mass loss rate for the classical T Tauri stars in their sample, relates mass accretion rate to total CaII line luminosity, and that it applies also at the lower masses (and radii) of the objects discussed here.
} 
Table 4. Outflow parameters derived from forbidden lines.

\begin{tabular}{lccccc}
\hline \hline Object & $\begin{array}{c}N_{\mathrm{e}} \\
\left(\mathrm{cm}^{-3}\right)\end{array}$ & $\begin{array}{c}L(\lambda 6300) \\
\left(L_{\odot}\right)\end{array}$ & $\begin{array}{c}L(\lambda 6731) \\
\left(L_{\odot}\right)\end{array}$ & $\begin{array}{c}\dot{M}(\lambda 6300)^{1} \\
\left(M_{\odot} \mathrm{yr}^{-1}\right)\end{array}$ & $\begin{array}{c}\dot{M}(\lambda 6731)^{1} \\
\left(M_{\odot} \mathrm{yr}^{-1}\right)\end{array}$ \\
\hline Par-Lup3-4 & 3000 & $2.3 \times 10^{-5}$ & $6.3 \times 10^{-6}$ & $8.0 \times 10^{-8}$ & $3.6 \times 10^{-8}$ \\
LS-RCrA 1 & 2300 & $2.8 \times 10^{-6}$ & $5.7 \times 10^{-7}$ & $1.2 \times 10^{-8}$ & $4.1 \times 10^{-9}$ \\
\hline
\end{tabular}

\footnotetext{
${ }^{1}$ Outflow mass loss rate estimated under the assumption of an outflow velocity of $150 \mathrm{~km} \mathrm{~s}^{-1}$ and an emitting region of size $9.1 \times 10^{14} \mathrm{~cm}$ (see text).
}

Besides Sz102, the most prominent emission in HeI is found in Sz113, a star having also intense $\mathrm{H} \alpha$ and CaII infrared triplet lines but no forbidden lines indicating outflows. The other objects displaying $\mathrm{HeI}$ emission also have strong $\mathrm{H} \alpha$ emission, with the exception of Sz110 where it is only moderate. Disk winds hot enough to produce $\mathrm{HeI}$ emission may be expected to produce also [OII] emission at 7319 and $7329 \AA$, which is observed only in LS-RCrA 1 and possibly Par-Lup3-4. For these reasons, we thus favor the base of the accretion columns as the formation site of the observed HeI emission.

The amplitudes observed in the VRI bands, as well as the rate at which they decrease towards longer wavelengths (see Sect. 3.1), are also expected as a consequence of accretion by the formation of hot spots in the surface. The origin of the near-infrared variability of Par-Lup3-4, although probably related also to accretion, may be somewhat different from that at visible wavelengths and related to changes in the inner regions of the accretion disk (Eiroa et al. 2002). Nearinfrared monitoring of other star forming regions (Kaas 1999; Carpenter et al. 2002) has shown that important variations in the $K$ band, typically around 0.3 mag but reaching up to 1 mag for some sources, is common among young stellar objects, with a trend for larger amplitudes to be associated with stronger near-infrared excesses.

\subsubsection{Outflow signatures and mass loss rates}

Forbidden lines of [OI], [NII], [SII], and [OII] are detected prominently in Sz102 and Par-Lup3-4, and much more faintly in Sz106. Ratios among some of these lines allow useful estimates of physical conditions in the circumstellar medium and mass loss rates through their dependencies with temperatures and densities (e.g. Osterbrock 1989). A difficulty nevertheless arises with the interpretation of our low resolution spectra, since forbidden lines in $\mathrm{T}$ Tauri spectra are usually split into two separate components with near-zero and high, generally blueshifted velocity respectively (e.g. Appenzeller et al. 1984). Besides the kinematical differences, both sets of lines sample different physical conditions (Hamann 1994). Unfortunately, due to lack of resolution in the spectra available for the objects discussed here it is not possible to carry out a separate analysis of the low- and high-velocity components. This is an important caveat at the time of interpreting the quantities estimated in this Section.

The ratio $[\mathrm{SII}](\lambda 6717) /[\mathrm{SII}](\lambda 6731)$, practically insensitive to the temperature, is often used to derive the electron density $N_{\mathrm{e}}$ in the emitting volume. The values we find for Sz102 and Par-Lup3-4 are very similar, also to those measured for LS-RCrA 1 (Table 4), and indicate $N_{\mathrm{e}}$ near $2000-3000 \mathrm{~cm}^{-3}$. These are indicative values only, due both to the blend of the low and high velocity components of those lines as pointed out above, and to the fact that they (especially the high velocity component) arise from an extended region (e.g. Cabrit et al. 1999) probing a wide range of densities.

Keeping the caveats about the components blend in mind, it is possible to estimate upper limits to the mass loss rates following the method outlined by Hartigan et al. (1995) based on the luminosities of the high velocity component of the [OI] $\lambda 6300$ or the $[\mathrm{SII}] \lambda 6731$ lines. For this purpose, we first derive the luminosity in the line from its measured equivalent width and from the dereddened $R_{0}$ magnitude of the object:

$L\left(L_{\odot}\right)=6.71 \times 10^{-5} D^{2}(\mathrm{pc}) E W(\AA) 10^{-0.4 R_{0}}$

where $D$ is the distance and $E W$ is the equivalent width of the line. We assume an extinction of $A_{R} \simeq 0$ for Sz102 and $A_{R}=0.37$ for LS-RCrA 1 (Fernández \& Comerón 2001). For Par-Lup3-4 we adopt $A_{R}=1.82$ corresponding to the lower value of the extinction, $A_{V}=2.4$, discussed in Sect. 3.4. A value closer to the upper one, $A_{V}=4.3$, would imply a much redder $\left(R_{\mathrm{C}}-I_{\mathrm{C}}\right)$ than observed in March 2002, from where we take $R$ used in Eq. (3). The derived mass loss rates are:

$$
\begin{aligned}
\dot{M}\left(M_{\odot} \mathrm{yr}^{-1}\right)= & 3.03 \times 10^{7}\left(1+\frac{N_{\mathrm{c}}}{N_{\mathrm{e}}}\right) \\
& \times \frac{L(\lambda 6300)\left(L_{\odot}\right) V\left(\mathrm{~km} \mathrm{~s}^{-1}\right)}{l(\mathrm{~cm})} \\
\dot{M}\left(M_{\odot} \mathrm{yr}^{-1}\right)= & 4.51 \times 10^{9}\left(1+\frac{N_{\mathrm{c}}}{N_{\mathrm{e}}}\right) \\
& \times \frac{L(\lambda 6731)\left(L_{\odot}\right) V\left(\mathrm{~km} \mathrm{~s}^{-1}\right)}{l(\mathrm{~cm})}
\end{aligned}
$$

from the $[\mathrm{OI}] \lambda 6300$ and $[\mathrm{SII}] \lambda 6731$ respectively, where $N_{\mathrm{c}}$ are the critical densities $\left(N_{\mathrm{c}}=2 \times 10^{6} \mathrm{~cm}^{-3}\right.$ for $[\mathrm{OI}] \lambda 6300$ and $N_{\mathrm{c}}=2 \times 10^{4} \mathrm{~cm}^{-3}$ for [SII] $\left.\lambda 6731\right), V$ is the outflow velocity, and $l$ is the linear size of the emitting region. These last two quantities are in principle unknown, since we do not possess kinematical information and all the sources appear unresolved in our observations. A rough estimate of the mass loss rate can be made however by assuming that the representative value of $V=150 \mathrm{~km} \mathrm{~s}^{-1}$ used by Hartigan et al. (1995) for higher mass $\mathrm{T}$ Tauri stars applies also to the objects studied here, and using the resolution of our images as an upper limit to the size of the emitting region. As noted by Fernández \& Comerón (2001), LS-RCrA 1 still appears unresolved in images with a full-width 
at half-maximum of only $0^{\prime \prime} 38$, corresponding to a linear scale of $\sim 9.1 \times 10^{14} \mathrm{~cm}(=60 \mathrm{AU})$ at the distance of the R CrA star forming region, which is the typical size of circumstellar disks suggesting that this is an acceptable approximation to the actual size of the emitting region. Adopting this value as an upper limit to the sizes of the emitting regions of the Lupus sources as well, we obtain mass loss rates of the order of $\sim 10^{-8} M_{\odot} \mathrm{yr}^{-1}$ for both Par-Lup3-4 and LS-RCrA 1. The values derived from [OI] $\lambda 6300$ and from [SII] $\lambda 6731$ are in agreement to a factor better than 3 , which is satisfactory enough for the estimates intended here.

It must be stressed however that these are upper limits to the mass loss rate, since we use the total equivalent width of the lines given the insufficient resolution to isolate the high velocity component - or even to establish its existence. Mass loss rates can be much lower if the low velocity component dominates the emission. Indications that this may indeed be the case come from the existing evidence for a correlation between the accretion (traced by infrared excess due to circumstellar emission or by veiling) and the mass loss processes (traced by forbidden lines), as has been discussed by Cabrit et al. (1990) among other works. In their comparison between outflow rates and accretion rates derived from spectral veiling, Hartigan et al. (1995) also find a loose correlation between both, the accretion rate being typically 10-100 times higher than the outflow rate. Taken at face value our results seem to depart considerably from this correspondence, as the mass loss rates discussed above are typically an order of magnitude greater than the accretion rates. We take this as an indication that actual mass loss rates are much smaller than the values given in Table 4, a hypothesis that could be easily confirmed if mid-resolution spectroscopy failed to reveal the blueshifted component on which the mass loss rate estimate is based. As a matter of fact, applying this derivation to Sz102 results in a mass loss rate of $3.5 \times 10^{-7} M_{\odot} \mathrm{yr}^{-1}$ versus an accretion rate 40 times smaller (which would result in its complete evaporation if maintained for only 1-2 Myr!). In that case however published medium resolution spectra (Appenzeller et al. 1984) fail to reveal any significant high velocity component, in consistency with a much lower mass loss rate.

In addition to $\mathrm{H} \alpha$, some of the upper lines of the Paschen series of hydrogen are visible in the spectra of Sz102, Sz106, and Par-Lup3-4 allowing the measurement of the Paschen decrement. Pa9, Pa10, Pa11, Pa12, and Pa 14 are clearly visible in $\mathrm{Sz}$ 102, while only $\mathrm{Pa} 9, \mathrm{~Pa} 10$, and Pa12 can be reliably measured in the other two objects. The ratios among these lines in Sz 102 and Sz 106 follow the expected values for recombination lines in a hot gas $\left(T \simeq 10^{4} \mathrm{~K}\right)$, which is only weakly dependent on temperature and density (Brocklehurst 1971). This is not the case however for Par-Lup3-4, whose Paschen decrement is noticeably slower: for example, the intensity ratio $\mathrm{Pa} 12$ / $\mathrm{Pa} 9$ is $\simeq 0.8$, about twice the expected value, and a similar behavior is seen for Pa10 / Pa9. This was also noticed by Eislöffel et al. (1990) in their comparison between T Tauri and AS 353A, the source powering HH 32, attributing the slower Paschen decrement of the latter to optically thick emission. The same conclusion may apply to Par-Lup3-4.

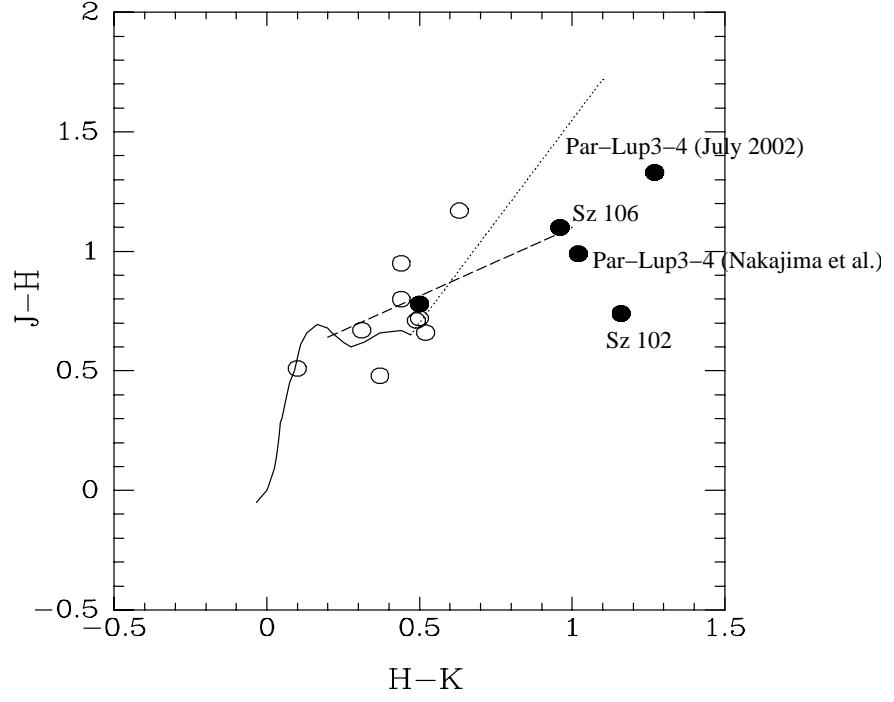

Fig. 4. Near-infrared color-color diagram displaying all the sources for which near-infrared photometry is available. The solid line represents the locus of unreddened main-sequence stars down to spectral type M6 (Bessell \& Brett 1988), and the dashed straight line is the classical T Tauri star locus derived by Meyer et al. (1997). The dotted line is the reddening vector representing the displacement of a star obscured by $A_{V}=10$ mag using the extinction law of Rieke \& Lebofsky (1985). Filled circles indicate sources with $E W(\mathrm{H} \alpha)>100 \AA$. Sources with infrared excess (to the right of the reddening vector) are indicated. ParLup3-4 is indicated twice, corresponding to the measurements presented by Nakajima et al. (2000) and in the present paper.

\subsubsection{Infrared excesses}

The accretion signatures in the visible among $\mathrm{T}$ Tauri stars and protostars ("Class II" and "Class I" sources respectively; Adams et al. 1987) are usually correlated with near- and midinfrared excesses indicating the existence of warm circumstellar material or the reprocessing of light from the central object by dust in a circumstellar envelope. This is clearly visible even in a small-size sample like the one discussed in this paper, as can be seen in Fig. 4. In it we plot the near infrared colors of all sources for which such information is available from Table 1, together with the locus occupied by normal mainsequence dwarfs (Bessell \& Brett 1988), by classical T Tauri stars (Meyer et al. 1997) and a reddening vector (Rieke \& Lebofsky 1985) whose length corresponds to $A_{V}=10 \mathrm{mag}$ and whose origin lies at the position of a dereddened M6 dwarf. Such a diagram provides an easy way of identifying sources with infrared emission of circumstellar origin, which are those lying to the right of the reddening vector as plotted in the figure (Lada \& Adams 1992).

The filled circles in Fig. 4 represent the sources with a $\mathrm{H} \alpha$ emission equivalent width exceeding $100 \AA$ A. Par-Lup3-4 is represented twice, corresponding to the $J H K$ photometry published by Nakajima et al. (2000) and the one obtained by us in July 2002. With the single exception of Sz 113, all the filled circles fall in the region denoting the existence of infrared excess. On the other hand, it is worth noticing that the $J H K$ photometry of LS-RCrA 1 published by Fernández \& Comerón (2001) places it at virtually the same position as Sz 113, indicating that 
the correlation between strong $\mathrm{H} \alpha$ emission and near-infrared excess suggested by Fig. 4 is not strict, and that strong emission lines relative to the continuum can be observed also among objects with no traces of near-infrared excess. It is interesting to notice also that all the stars with large $\mathrm{H} \alpha$ equivalent widths, with the exception of the extreme case of $\mathrm{Sz} \mathrm{102,} \mathrm{lie} \mathrm{close} \mathrm{to}$ the unreddened locus of classical T Tauri stars.

\subsection{4. $\mathrm{H} \alpha$ intensities and continuum fluxes}

One of the most interesting features of the present sample is that the two sources with the largest $\mathrm{H} \alpha$ equivalent width, Sz 102 and Par-Lup3-4, are also the faintest ones at infrared wavelengths. $\mathrm{Sz} 102$ is also the bluest in $\left(R_{\mathrm{C}}-I_{\mathrm{C}}\right)$, which is partly due to the intense emission lines that dominate over the photospheric emission in the $R$ band (inducing in the case of Sz 102 a bluening of $0.45 \mathrm{mag}$ in $\left.\left(R_{\mathrm{C}}-I_{\mathrm{C}}\right)\right)$ and partly due to intense veiling. The bluening in $\left(R_{\mathrm{C}}-I_{\mathrm{C}}\right)$ due to the emission lines in the $R$ band also affects noticeably the colors of Sz 106 (0.08 mag), Sz 113 (0.09 mag), Par-Lup3-4 (0.37 mag), and LS-RCrA 1 ( $0.31 \mathrm{mag})$. The faintness of Par-Lup3-4 may be partly explained by its late spectral type, but the sample contains at least 7 objects that are both later and much brighter, including the latest object of the sample that we estimate to be 2.5 subclasses later. We note that Sz113, which we classify as M6, also has strong $\mathrm{H} \alpha$ and CaII emission and is over one magnitude fainter than the rest of the objects of similar spectral type at $I_{\mathrm{C}}$ and $J$. These differences tend to maintain themselves or decrease only slowly as we move towards longer wavelengths, contrarily to what would be expected if they were due to different amounts of extinction along the lines of sight towards each object.

It must be noted that the truly anomalous property of the sources with large $\mathrm{H} \alpha$ equivalent widths is the low luminosity of the underlying continuum, rather than the intrinsic intensity of the $\mathrm{H} \alpha$ emission. A comparison of the quantity $2.5 \log (E W(\mathrm{H} \alpha))-R_{\mathrm{C}}+D M$, which provides a measure of the absolute intensity of the $\mathrm{H} \alpha$ emission, shows that Par-Lup3-4 and LS-RCrA 1 display $\mathrm{H} \alpha$ intensities similar to those of Sz 109, Par-Lup3-2, and Par-Lup3-3, which are in the same spectral type range but have normal luminosities. Sz 100, Sz 112, and Sz 114, having spectral types M5-M6, have higher $\mathrm{H} \alpha$ fluxes but no signs of underluminosities.

\subsection{Temperature-luminosity diagram}

To better quantify the apparent underluminosity of the sources with the largest $\mathrm{H} \alpha$ equivalent widths, we have followed the same procedure as outlined by Fernández \& Comerón (2001) to place all the M-type objects of our sample in a temperatureluminosity diagram enabling comparison to pre-main sequence evolutionary tracks. The estimate of the extinction $A_{V}$ towards each source is made by comparing the $(J-H)$ color to an assumed intrinsic $(J-H)_{0}$ using the Rieke \& Lebofsky (1985) extinction law, which gives $A_{V}=9.36\left[(J-H)-(J-H)_{0}\right]$. For the intrinsic colors we take as a base those of Bessell \& Brett (1988), to which we add $\Delta(J-H)_{0}=+0.11$ mag in order to match the colors adopted at spectral type M6 by Comerón et al. (2000) for the Chamaeleon I population. Such offset is intended to roughly account for the redder intrinsic colors of young, lower surface gravity objects at a given spectral type, as described by Comerón et al. (2000). The values of $A_{V}$ calculated in this way are thus 1.0 mag lower than those that would be obtained directly using Bessell \& Brett's colors with no further correction. Even lower values, closer to zero, are obtained by dereddening the positions of the objects in Fig. 4 to the position of the T Tauri star locus, in which $(J-H)_{0}$ is higher, although the applicability of this line to objects as late as the ones discussed here may be questioned. The results are given in Table 5. We have adopted $A_{V}=0$ for those sources for which we obtain negative values of the extinction. In general such negative values do not exceed $0.6 \mathrm{mag}$ and may be attributed to errors in the photometric measurement or in the assumed intrinsic colors. The exceptions are Sz 109 and Par-Lup3-2, for which we obtain $A_{V}=-2.7$ and -2.4 , respectively. We also adopt $A_{V}=0$ for Par-Lup3-1, consistent with its visible colors and its location in a zone of low extinction on the background.

The reader is referred to Fernández \& Comerón (2001) for a discussion on the temperature scale and bolometric corrections that we also use here. We note that, although systematic differences may exist between the adopted and the actual values of temperatures and bolometric corrections, such differences should affect in a similar manner all the objects under consideration and thus should have virtually no impact on the evaluation of the underluminosity on which we focus our discussion. On the other hand, the choice of the $J$ band as the reference for the derivation of the luminosity reduces the impact of possible errors in the derivation of the extinction, which we obtain using the $(J-H)$ color, and of the possible contribution of circumstellar material to the flux. We have excluded from this discussion Sz102, whose spectrum is likely to be heavily veiled due to accretion, thus making the spectral classification very uncertain. In any case, it is most probably earlier than $\mathrm{M}$ and possibly close to the $\mathrm{K} 0$ spectral type assigned to it by Hughes et al. (1994).

In the case of Par-Lup3-4, the strong variability in infrared colors adds an extra difficulty to the estimate of extinction and luminosity. The value of $A_{V}$ obtained from the $(J-H)$ color in Table 1 is 5.6, but it reduces to 2.4 if the colors of Nakajima et al. (2000) are adopted. However, the higher derived extinction when the object is fainter and redder turns out to produce a difference of only $0.21 \mathrm{mag}$ in the dereddened magnitude $J-A_{J}$, thus making the luminosity estimate little dependent on the chosen set of $J H$ magnitudes.

The temperature-luminosity diagram is shown in Fig. 5 where, to further illustrate the point, we have also included the position of LS-RCrA 1, taken directly from Fernández \& Comerón (2001). Overplotted in that figure are the pre-main sequence evolutionary tracks of Baraffe et al. (1998) that may be used in principle to estimate the age and mass of each object. All the objects with $E W(\mathrm{H} \alpha)<100 \AA$ tend to lie in a small range of ages below $2 \mathrm{Myr}$ (note that the precise value depends on the adopted distance, as discussed in Sect. 2.3). LS-RCrA 1 and Par-Lup3-4, the objects with the most intense emission lines in the diagram, fall significantly below the isochrones 


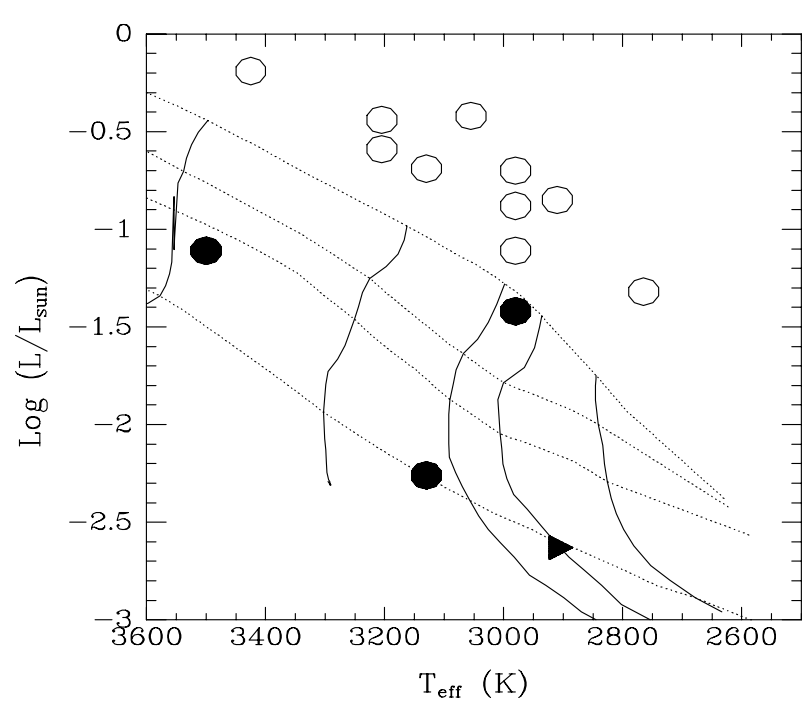

Fig. 5. Temperature-luminosity diagram showing the positions of the objects listed in Table 2, with the exception of Sz102. The superimposed curves are the pre-main sequence evolutionary tracks of Baraffe et al. (1998). As discussed in Sect. 2.3, a distance of $200 \mathrm{pc}$ has been adopted for the Lupus 3 star forming cloud. Full circles indicate $\mathrm{H} \alpha$ equivalent widths greater than $100 \AA$. For comparison, the position of LS-RCrA 1 is also plotted in this diagram (triangle). The uncertainties in $T$ and $\log L$ are approximately $\pm 400 \mathrm{~K}$ and $\pm 0.2 \mathrm{dex}$ respectively, and are dominated by uncertainties in the spectral typetemperature calibration and the distance, respectively; thus, the relative positions of the objects in the diagram are largely unaffected by such uncertainties.

corresponding to the ages around $10 \mathrm{Myr}$ or less that should be expected for the members of this star forming region. A similar but less marked trend is seen in Sz106 and Sz113, the next objects in order of decreasing $\mathrm{H} \alpha$ equivalent width. This trend is further illustrated in Table 5, where we list the masses and ages obtained by using the evolutionary tracks of Baraffe et al. (1998). These values are only indicative of the true physical parameters of the observed objects, given the sources of uncertainty involved in evolutionary calculations at such early ages (Wuchterl 2001; Baraffe et al. 2002) even regardless of the effect of accretion. The correlation between $\mathrm{H} \alpha$ equivalent width and derived age (in a direction which is furthermore the opposite from what would be expected if younger objects had higher accretion rates) and the derivation of unrealistic ages for members of a star forming region having extreme $\mathrm{H} \alpha$ equivalent widths obviously calls for a cautionary use of pre-main sequence evolutionary models when evaluating the physical parameters of such objects. We thus consider that the values given in Table 5 are reliable only for objects having $\mathrm{H} \alpha$ equivalent widths below $\sim 100 \AA$. It should be noted that one of such objects, Par-Lup3-1, is a bona fide brown dwarf judging from the derived mass and from its M7.5 spectral type together with its young age.

\subsubsection{X-ray luminosities}

Deep pointed observations of Lupus 3 in the ROSAT archive obtained with the Position-Sensitive Proportional Counter
Table 5. Derived masses and ages using the Baraffe et al. (1998) evolutionary tracks

\begin{tabular}{lccc}
\hline \hline Object & $A_{V}$ & Mass $\left(M_{\odot}\right)$ & Age (Myr) \\
\hline Sz100 & 0 & 0.2 & $\sim 1.5$ \\
Sz106 & 3.3 & 0.45 & 18 \\
Sz108A & 0 & 0.5 & $\sim 1.5$ \\
Sz108B & 0 & 0.1 & $\sim 1.5$ \\
Sz109 & 0 & 0.08 & $\sim 1$ \\
Sz110 & 2.2 & 0.25 & $\sim 1.5$ \\
Sz112 & 0 & 0.12 & $\sim 1$ \\
Sz113 & 0.1 & 0.09 & 3 \\
Sz114 & 0.5 & 0.15 & $\sim 1$ \\
Par-Lup3-1 & 0 & 0.04 & $\sim 1$ \\
Par-Lup3-2 & 0 & 0.11 & $\sim 1$ \\
Par-Lup3-3 & 4.3 & 0.3 & 50 \\
Par-Lup3-4 & 5.6 - 2.4 & 0.12 & \\
\hline Note: &
\end{tabular}

(PSPC) include all the objects discussed in this paper. Merging together all the existing PSPC datasets (200348p, 200351p, 200358p, 200359p, 200736p, 201209p, 201210p, 201211p, 201213p, 201213p-1, and 201276p) which amount to approximately $15 \mathrm{ksec}$ of integration time, it is thus possible to obtain detections or upper limits for our sources in order to study the correlation between the X-ray luminosity and the bolometric luminosity or other signs of activity. We used the EXSAS source detection routine running under MIDAS to perform local and map maximum likelihood source detection as described in Comerón et al. (2000), and considered association with a visible source if the positional coincidence between the optical and the X-ray position is better than 40". In addition, $\mathrm{Sz} 108$ (both components unresolved) is detected in a shorter integration pointing of the ROSAT HRI (High Resolution Imager; dataset 201649h) within $3^{\prime \prime}$ from the optical position.

Besides the HRI detection of Sz 108, only Sz 112 is detected in the ROSAT observations. A strong detection is obtained within the PSPC error box of Sz 106, but the nominal X-ray position is closer to HR 5999 than to $\mathrm{Sz} 106$ and we thus consider than an association with the much brighter star HR 5999 is far more likely. For all the other objects only upper limits to the X-ray luminosity are obtained. To convert the observed fluxes or upper limits to X-ray luminosity, we used a conversion factor of $10^{-11}$ counts $\mathrm{cm}^{2} \mathrm{erg}^{-1}$ based on the assumption that the X-ray emission can be approximated by a typical T Tauri-like Raymond-Smith spectrum with a plasma temperature of $1 \mathrm{keV}$, as justified in Comerón et al. (2000), corrected for the approximate value of the extinction derived as described in Sect. 3.4.

The X-ray luminosities and upper limits are given in Table 6, together with the maximum likelihood parameter, $M L$, for the two detected sources (where $M L=5$ corresponds to a $\sim 3 \sigma$ detection). The upper limits are generally very close to the normal level of emission from very low mass young stars and 
Table 6. X-ray luminosities.

\begin{tabular}{lcc}
\hline \hline Object & $M L$ & $\log \left(L_{\mathrm{X}} / L_{\mathrm{bol}}\right)$ \\
\hline Sz 100 & $<-4.13$ \\
Sz 102 & - \\
Sz 106 & - \\
Sz 108 $(\mathrm{A}+\mathrm{B})^{3}$ & 11.3 & -3.91 \\
Sz 109 & $<-3.99$ \\
Sz 110 & $<-3.64$ \\
Sz 112 & 39 & -4.08 \\
Sz 113 & $<-2.90$ \\
Sz 114 & $<-4.25$ \\
Par-Lup3-1 & $<-3.39$ \\
Par-Lup3-2 & $<-3.88$ \\
Par-Lup3-3 & $<-4.05$ \\
Par-Lup3-4 & $<-2.39$ \\
\hline Notes: & \\
${ }^{1} \log \left(L_{\mathrm{X}} / L_{\odot}\right)<-4.83 ;$ in this case, $L_{\text {bol }}$ is poorly determined. \\
${ }^{2}$ Confusion with nearby HR 5999. \\
${ }^{3}$ HRI observation.
\end{tabular}

to those of the actually detected objects $\left(\log \left(L_{\mathrm{X}} / L_{\mathrm{bol}}\right) \simeq-4\right)$, suggesting that a somewhat deeper integration may add some more detections.

The relatively high upper limits that we find for Sz 113 and Par-Lup3-4 are due to their low bolometric luminosities, and would be similar to those found for the other objects if their bolometric luminosities were replaced by those of objects with weak $\mathrm{H} \alpha$ of the same spectral type in the region. This may not be true however for Sz 102: if its luminosity were replaced by that of a $\sim 1 \mathrm{Myr}$-old K-type star, its non-detection implies $\log \left(L_{\mathrm{X}} / L_{\mathrm{bol}}\right)<-5$, or about one order of magnitude below the expected level.

\section{Discussion}

\subsection{Accretion-modified evolution, edge-on disks, or embedded sources?}

Par-Lup3-4 and LS-RCrA 1 seem to be so far the only examples of mid-M type objects with such strong emission lines relative to an unusually faint photospheric continuum. Equivalent widths of several hundred $\AA$ have been reported in the last years also for a number of very late-type members of starforming regions with no traces of underluminosity (Martín et al. 2001; Briceño et al. 2002; Zapatero Osorio et al. 2002; Barrado y Navascués et al. 2002; Luhman et al. 2003). All those cases correspond to objects with spectral types later than M7, and inspection of the published spectra shows that the large equivalent widths are due to the decreasing flux in the local (pseudo-) continuum at $\mathrm{H} \alpha$ rather than to the strength of $\mathrm{H} \alpha$, the $L(\mathrm{H} \alpha) / L_{\mathrm{bol}}$ ratio being several times smaller than for Par-Lup3-4 and LS-RCrA 1. However, it is worth mentioning the recently reported case of KPNO Tau 12 (Luhman et al. 2003), a M9 young brown dwarf with strong $\mathrm{H} \alpha$ emission as well as detectable $\mathrm{HeI}$ and $\mathrm{CaII}$ triplet emission, whose position in the temperature-luminosity diagram also falls significantly below the isochrones around which other young members of the Taurus clouds tend to appear.

The other object with extreme $\mathrm{H} \alpha$ emission in our sample (in this case concerning both relative and absolute intensity of the emission lines) is Sz 102, whose apparent underluminosity given its spectral type was already pointed out by Hughes et al. (1994). These authors noted that the geometry of its associated outflow (Krautter 1986) was suggestive of an edge-on disk perpendicular to it. Hughes et al. thus suggested that the reason for the apparent underluminosity of Sz 102 could be due to the fact that we may be seeing it in scattered light, rather than directly. Many other young stellar objects surrounded by edge-on disks are currently known (e.g. Burrows et al. 1996; McCaughrean \& O'Dell 1996, Stapelfeldt et al. 1998; Woitas \& Leinert 1998, Monin \& Bouvier 2000; Brandner et al. 2000; Jayawardhana et al. 2002; Grosso et al. 2003) or suspected (Martín 2000).

\subsubsection{Edge-on disks}

Can the underluminosity of the Lupus sources discussed here, LS-RCrA 1, and KPNO Tau 12 be also due to a particular viewing geometry rather than to the intrinsic properties of the object? In principle, an edge-on disk may enhance the intensity of emission lines by blocking the light from the central object if they formed in a region well detached from the photosphere, not occulted by the circumstellar disk, so that even moderate emission-line luminosities may produce large equivalent widths when superimposed on a photospheric continuum seen only in scattered light. Evidence for such an extended emitting region is indeed provided by intense forbidden lines in both Par-Lup3-4 and LS-RCrA 1, as discussed in Sect. 3. However, the excitation conditions of the gas in the extended, low-density regions of the disk wind set a limit to how much $\mathrm{H} \alpha$ flux can be contributed by them. Indeed, as noted by Fernández \& Comerón (2001) the $\mathrm{H} \alpha /[\mathrm{SII}]$ ratio in LS-RCrA 1 is 11 , far above the value of 4 that is found in the highest excitation Herbig-Haro objects. A lower, but still high ratio of 6.7 is measured in Par-Lup3-4, similar to that of Sz 102. The ratios are much higher for the other objects displaying both a certain degree of underluminosity and strong $\mathrm{H} \alpha$ emission, Sz 106 and Sz 113, where forbidden lines are either faint or not detected at all. Interestingly, the situation is different for two well-known objects having confirmed edge-on disks, HH30 (Cohen \& Jones 1987) and HV Tau C (Monin \& Bouvier 2000), indicating that in those two cases the complete emission-line spectrum, including $\mathrm{H} \alpha$, can be explained by the disk wind only. On the opposite, it appears that, for all the objects discussed here, most or nearly all of the $\mathrm{H} \alpha$ emission is contributed by a high excitation, high density emitting region that must lie closer to the object such as the accretion columns extending up to the inner edge of the truncated accretion disk only a few object radii away, or the basis of a jet.

While $\mathrm{H} \alpha$ emission is the most easily observable indicator of inflow and outflow activity, it is also a rather poor quantitative indicator of those phenomena due to the broad diversity of environments in which $\mathrm{H} \alpha$ emission is produced. Stronger 
constraints are provided by the CaII and HeI lines, whose origin in accretion near the object surface is much better established (e.g. Alencar et al. 2001), and whose origin in outflows would be harder to account for in view of the high temperatures and mass loss rates needed (Hartmann et al. 1990; Beristain et al. 2001). For the CaII infrared triplet lines in our objects, their ratios close to 1:1:1 indicate that they are formed near the photosphere of the central object rather than in an extended low density envelope (Graham \& Heyer 1988). Observational evidence for the intimate connection between these lines and the accretion process is provided by the observed correlation between variations in the red wing of $\mathrm{H} \alpha$ and the strength of the CaII broad component in BP Tau (Gullbring et al. 1996) or by the correlation between the strengths of CaII and HeI in time series observations of DF Tau (Johns-Krull et al. 1997), besides the already noted correlation between veiling and CaII emission investigated by Muzerolle et al. (1998b) that we used in Sect. 3.3.1 to derive accretion rates. Also the appearance of HeI emission, needing high excitation temperatures that can probably happen only at the bases of the accretion columns, argues for its origin near the surface of the object.

Since these lines most likely form in the immediate vicinity of the central object, they should be also obscured by a possible edge-on disk if that were the cause of the underluminosity. The prominence of these lines in Sz 113, Par-Lup3-4, and LSRCrA 1 thus indicates that they are intrinsically intense as compared to the underlying continuum. In other words, our data argue in favor of intrinsically high $L_{\mathrm{em}} / L_{\mathrm{bol}}$ ratios (where $L_{\mathrm{em}}$ and $L_{\text {bol }}$ are respectively the luminosity of any of the emission lines formed near the surface of the object and the bolometric luminosity) for the objects considered here, regardless of whether or not they are occulted by edge-on disks.

The arguments discussed in the previous paragraph in favor of intrinsically high line-to-continuum ratios only show that such ratios may not be due to partial blocking by edge-on disks, but in principle does not rule out the possibility that the overall underluminosity may be due to edge-on disks blocking both the continuum and the line formation region. If this were the cause, normally luminous objects with similar emission line to bolometric luminosity ratios should be much more common (and brighter, hence easier to identify) than the underluminous ones, since the latter would be only the result of a particular viewing geometry. However, the fact that no late-type objects with such extreme emission line spectra and normal luminosity have been identified leads us to consider it highly unlikely that edge-on disks are the cause of the phenomena described here.

\subsubsection{Embedded Class I sources}

Another interesting possibility is that the apparently underluminous objects are actually fully embedded in a thick circumstellar envelope and undergoing the very high accretion and mass outflow rates characteristic of Class I sources, which would naturally produce the large emission-line to continuum ratios. Class I objects with spectral types only slightly earlier than those of Par-Lup3-4 or LS-RCrA 1 and very similar emission-line spectra have been observed in the visible by Kenyon et al. (1998). The observed emission does not come in this case directly from the central star, which is hidden from view by extinctions in the $A_{V}=30-60 \mathrm{mag}$ range. Instead, the star is seen via the light scattered by the walls of cavities in the envelope having a relatively unimpeded line of sight toward the central star. This indirect view of the star through scattered light would in turn explain the apparent underluminosities. Some of the spectra presented by Kenyon et al. (1998), in particular those of HH31 IRS2 and 04158+2805, bear indeed a great resemblance to those of Par-Lup3-4 or LS-RCrA 1, although without the extreme relative emission line strengths that we measure in our sources.

However, a distinctive characteristic of optically visible Class I sources is that they are spatially extended and resolved at the distance of nearby star forming regions. Kenyon et al. (1998) find optical sizes normally in the $5^{\prime \prime}-10^{\prime \prime}$ range for their Taurus sources, which would be easily resolved also in our Lupus images despite of the somewhat larger distance. These are also typical sizes expected from models of the appearance of circumstellar envelopes in the near-infrared (Whitney et al. 1997). However, none of our underluminous sources, including LS-RCrA 1, shows any hints of resolved circumstellar nebulosity. The image quality of our observations of Lupus 3 is not very good (FWHM of 1" 4 in both our visible and nearinfrared images), but should suffice to resolve nebulosity of the same physical size as that of the Taurus sources. Much more constraining are our near-infrared observations of LS-RCrA 1, as noted in Sect. 3.3.2, which allow to set an upper limit of only $0^{\prime \prime} 38$ (60 AU) to the extent of any possible infrared nebula around this object. As noted by Whitney et al. (1997), the existence of extended nebulosity is an unambiguous criterion for the distinction between Class I and Class II sources, and none of our objects can be classified as Class I according to it.

Class I sources also tend to have conspicuous near-infrared excesses, as confirmed by both observations and models (Whitney et al. 1997). As discussed in Sect. 3.3.3, we do see a trend among sources with high $\mathrm{H} \alpha$ equivalent widths towards having near-infrared excesses (see also Fig. 4). However, leaving aside the most extreme case of $\mathrm{Sz} \mathrm{102,} \mathrm{two} \mathrm{of} \mathrm{our}$ sources (Par-Lup3-4 and Sz 106) have colors significantly bluer than the average of the Whitney et al.'s sample, and two other (Sz 113 and LS-RCrA 1) display no signs of infrared excess at all. Moreover, as noted in Sect. 3.3.3, all the late-type sources with large $\mathrm{H} \alpha$ equivalent widths occupy positions in the $(J-H)$, $(H-K)$ diagram close to the locus of unreddened classical T Tauri stars (class II objects) as observed by Meyer et al. (1997). Such lack of strong infrared excess is reproduced in the Class I object models of Whitney et al. only when the line of sight is approximately coincident with the axis of the dust-free bipolar cavities, thus providing a nearly unobscured view of the central object. However, reproducing the underluminosity that we observe among our sources does require a significant amount of obscuration, and therefore most probably redder infrared colors than the ones that we observe.

Although the available imaging observations do not favor the interpretation of the observed features as due to a possible Class I nature of the objects under discussion, it is interesting 
to note that a more definitive proof could be provided by polarimetric observations offering the possibility of probing the properties of circumstellar envelopes (Whitney et al. 1997).

\subsubsection{Accretion-induced underluminosity}

A third possibility to be considered is that the evolution of our sources along their convective tracks may have been substantially modified by accretion. The impact of sustained accretion on a limited area of the stellar surface after reaching the birthline (Stahler 1983) has been studied by Hartmann et al. (1997; see also Siess et al. 1997). The results for stars with initial masses of $0.3 M_{\odot}$ and above show that the luminosity evolution of a star is accelerated by accretion, in a way such that the star appears in the $T_{\text {eff }}-L$ diagram at the position that a non-accreting star with the same instantaneous mass, but an older age, would have. Observationally, the difference between accreting and non-accreting stars at a given spectral type is that the former started their post-birthline evolution with a lower mass, and are at a given time less luminous than the non-accreting ones. While this is a good qualitative description of what is actually observed in our sample, the magnitude of the observed effect is huge (in terms of difference in $L$ at a given $\left.T_{\text {eff }}\right)$ compared to the quantitative effects estimated by Hartmann et al. (1997). Given the reasonable assumptions of that work regarding the treatment of accretion, it seems very difficult with such an approach to reach the level of the observed effects. However, Hartmann et al. limited their study to initial masses larger than $0.15 M_{\odot}$. As Table 5 indicates, the initial masses at the birthline of the objects that we discuss here may be far below that value. The effect of accretion may conceivably be more dramatic for such low initial masses closer to the brown dwarf regime, even with the moderate (in absolute terms) accretion rates that we estimate in Sect. 3.3.1. Given the present data and uncertainty of mass estimates, this conclusion remains speculative and deserves an extension of the work by Hartmann et al. (1997) to lower masses. This work is under progress. On the other hand, we must note in this context the intriguing non-detection in X-rays of the most extreme emissionline object in the region, $\mathrm{Sz} 102$, which suggests that its underluminosity in $L_{\text {bol }}$ is corresponded by an underluminosity in $\mathrm{X}$-ray emission as compared to typical low mass objects of the same age. Such correspondence can be readily explained by an edge-on disk or a circumstellar envelope that should also attenuate the X-ray emission produced by chromospheric activity in the central object, while it is less straightforward to explain how accretion-induced underluminosity may also decrease the level of X-ray emission. An X-ray detection (or a more strict upper limit) of Par-Lup3-4 or LS-RCrA 1 would be valuable, as it would decide whether or not Sz 102 may be regarded as a special case.

\subsection{Impact on statistics of young stellar aggregates}

What may be the implications of the existence of such low mass underluminous objects on the study of the mass and age distributions in star forming regions? As Fig. 5 shows, if our favored hypothesis of accretion-modified evolution is the correct one to explain the existence of such underluminous objects, existing evolutionary tracks that do not include accretion are of little use for the derivation of the physical parameters of such objects, in agreement with the cautionary advise of Hartmann et al. (1997) on the interpretation of ages in star forming regions. These objects are easy to exclude from the samples used to study the mass function and age distribution of star forming regions provided that spectroscopy is available. They may represent however a contamination in samples selected on the bases of their near-infrared colors for which spectroscopy in the visible is not available, a frequently encountered case and an unavoidable one when studying heavily embedded aggregates. The scarcity of very low mass objects with $\mathrm{H} \alpha$ equivalent widths much above $100 \AA$, such as Par-Lup3-4 and LSRCrA 1, suggests in principle that such contamination may not be very important. Nevertheless, the possibility that the effects of strong accretion during early evolution may remain after accretion has ceased needs to be taken into account. It should be noted in this context that temperature-luminosity diagrams of star forming regions often show outliers falling well below the band defined by the bulk of the members, but whose spectral characteristics are otherwise undistinguishable from those of apparently younger members of the same spectral type. The explanation of such outliers is unclear, and in general it may not be excluded that they are the result of an earlier episode of star formation. However, in the light of the results presented here, we offer the alternative explanation that they may actually be post-accretion objects.

\section{Summary and conclusions}

In this paper we have reported on new slitless and multi-object spectroscopy observations of the central area of the Lupus 3 star forming cloud in a search for new members characterized by their $\mathrm{H} \alpha$ emission. The observations have revealed four previously unidentified members having spectral types between M4.5 and M7.5, the latter one being a brown dwarf.

The spectra of previously known and new members present a wide range of $\mathrm{H} \alpha$ equivalent widths reaching up to $430 \AA$. The new object with the largest $\mathrm{H} \alpha$ equivalent width, Par-Lup34, displays a rich emission-line spectrum indicating both accretion and mass loss, as well as strong variability in both brightness and color in the near-infrared and moderate near-infrared excess. The strength of the emission lines and the late spectral type of the underlying continuum make it very similar to a previously reported object, LS-RCrA 1, the only other mid-M type object displaying such intense emission-line spectra identified so far. Even more remarkably, Par-Lup3-4 shares with LS-RCrA 1 the property of being much less luminous than objects of their spectral types without such prominent emissionline spectra and a few million years of age. The same seems to apply to Sz 102, although its featureless spectrum in the range covered here precludes its classification. Noticeable underluminosity, although not so extreme, is also found among other objects in our sample with $\mathrm{H} \alpha$ equivalent widths exceeding $100 \AA$ A Sz 106 and Sz 113. A comparison among the $\mathrm{H} \alpha$ fluxes shows that the reason for the large $\mathrm{H} \alpha$ equivalent widths 
is the faintness of the underlying continuum, rather than the absolute intensity of the emission lines, except in the case of $\mathrm{Sz} 102$ that is also the strongest $\mathrm{H} \alpha$ emitter of our sample.

Based on the correlation between CaII emission and mass accretion rate, we estimate accretion rates between $1.0 \times 10^{-9}$ and $1.9 \times 10^{-8} M_{\odot} \mathrm{yr}^{-1}$ for the objects in our sample. An upper limit of several times $10^{-8} M_{\odot} \mathrm{yr}^{-1}$ is found for the mass loss rate of Par-Lup3-4, although much lower values are possible in the likely case that the forbidden-line emission is dominated by a low velocity component.

We have considered different possible causes for the underluminosity of objects with large $\mathrm{H} \alpha$ equivalent widths. The strength of $\mathrm{H} \alpha$, the $\mathrm{CaII}$ infrared triplet, and $\mathrm{HeI}$ with respect to the forbidden line spectrum leads us to the conclusion that most of the $\mathrm{H} \alpha$ luminosity is probably contributed by high density regions near the surface of the star, and that the high $L(\mathrm{H} \alpha) / L_{\mathrm{bol}}$ is an intrinsic feature of these objects rather than a geometric effect caused by partial obscuration by an edge-on disk. We also find it unlikely that these objects are embedded Class I sources seen through scattered light, due to the lack of spatially extended nebulosity and to the moderate or non-existent infrared excesses among them. A third possibility is that these objects started their post-birthline evolution at very low, probably substellar masses, and that further accretion has modified their evolution making them appear at present much older than they actually are given their mass. This effect is suggested, although with less dramatic effects, by evolutionary models incorporating accretion at higher masses, and an extension of those works to the substellar range may be needed to confirm that it is an adequate explanation in the cases discussed here. We speculate that the members of star forming regions sometimes found with apparent ages well above those of most of the other members might actually be post-accretion objects.

Two of the previously known objects, Sz 108 and Sz 112, are detected in X-rays with emission levels similar to those observed in low mass stars in other star forming regions. Upper limits available for the other sources discussed in this paper are also consistent with those levels, although only in one case (Sz 102) does the upper limit suggests that the global underluminosity is corresponded by an underluminosity also in X-ray emission.

The detailed modelling of $\mathrm{T}$ Tauri stars that high resolution spectroscopy and time monitoring have made possible calls for a similar observational effort focused on objects such as those described here. Also, the distance to regions such as Lupus and R CrA may allow the resolution of circumstellar disks by adaptive optics imaging, and therefore a direct test of the possibility that underluminosity may be caused by edge-on disks. Another attractive observational approach is polarimetry, which offers the possibility of probing the existence and geometry of circumstellar envelopes. If the underluminosity turned out to be real, as we propose in this work, the observations of these objects will provide important constraints to new generations of pre-main sequence evolutionary models including accretion.

Acknowledgements. We are pleased to thank the staff of Paranal Observatory for their support during our observations, especially Drs. Hermann Böhnhardt and Olivier Marco. We are particularly thankful for their acceptance of our request for a change of instrument coordinated with another program of ours scheduled during the same nights, despite of the increased support effort involved. The support received at the La Silla Observatory by the $2.2 \mathrm{~m}$ and the NTT Teams, and in particular Drs. Lisa Germany, John Pritchard, Malvina Billères, and Olivier Hainaut, is also gratefully acknowledged. Extensive and constructive comments by an anonymous referee led to substantial improvements in the contents of this paper. IB acknowledges discussions with Lee Hartmann and Kevin Luhman on the edge-on disk hypothesis. MF has been partially supported by the Spanish grant AYA2001-1696. RN wishes to acknowledge financial support from the Bundesministerium für Bildung und Forschung through the Deutsche Zentrum für Luft- und Raumfahrt e.V. (DLR) under grant number 50 OR 0003 .

\section{References}

Adams, F. C., Lada, C. J., \& Shu, F. H. 1987, ApJ, 312, 788

Alencar, S. H. P., Johns-Krull, C. M., \& Basri, G. 2001, AJ, 122, 3335

Appenzeller, I., Jankovics, I., \& Krautter, J. 1983, A\&AS, 53, 291

Appenzeller, I., Jankovics, I., \& Östreicher, R. 1984, A\&A, 141, 108

Baraffe, I., Chabrier, G., Allard, F., \& Hauschildt, P. H. 1998, A\&A, 337,403

Baraffe, I., Chabrier, G., Allard, F., \& Hauschildt, P. H. 2002, A\&A, 382,563

Barrado y Navascués, D., Zapatero Osorio, M. R., Martín, E. L., et al. 2002, A\&A, 393, L85

Batalha, C. C., Stout-Batalha, N. M., Basri, G., \& Terra, M. A. O. 1996, ApJS, 103, 211

Beristain, G., Edwards, S., \& Kwan, J. 2001, ApJ, 551, 1037

Bessell, M. S. 1979, PASP, 91, 589

Bessell, M. S., \& Brett, J. M. 1988, PASP, 100, 1134

Blaauw, A. 1991, in The Physics of Star Formation and Early Stellar Evolution, ed. C. J. Lada, \& N. D. Kylafis (Kluwer)

Brandner, W., Sheppard, S., Zinnecker, H., et al. 2000, A\&A, 364, L13

Briceño, C., Luhman, K. L., Hartmann, L., Stauffer, J. R., \& Kirkpatrick, J. D. 2002, ApJ, 580, 317

Brocklehurst, M. 1971, MNRAS, 153, 417

Burrows, C. J., Stapelfeldt, K. R., Watson, A. M., et al. 1996, ApJ, 473, 437

Cabrit, S., Edwards, S., Strom, S. E., \& Strom, K. M. 1990, ApJ, 354, 687

Cabrit, S., Ferreira, J., \& Raga, A. C. 1999, A\&A, 343, L61

Carpenter, J. M., Hillenbrand, L. A., Skrutskie, M. F., \& Meyer, M. R. 2002, AJ, 124, 1001

Chen, H., Grenfell, T. G., Myers, P. C., \& Hughes, J. D. 1997, ApJ, 478, 295

Cohen, M., \& Jones, B. F. 1987, ApJ, 321, 846

Comerón, F., Neuhäuser, R., \& Kaas, A. A. 2000, A\&A, 359, 269

Eiroa, C., Oudmaijer, R. D., Davies, J. K., et al. 2002, A\&A, 384, 1038.

Eislöffel, J., Solf, J., \& Böhm, K. H. 1990, A\&A, 237, 369

Fernández, M., \& Comerón, F. 2001, A\&A, 380, 264

Fukugita, M., Ichikawa, T., Gunn, J. E., et al. 1996, AJ, 111, 1748

Graham, J. A., \& Heyer, M. H. 1988, PASP, 100, 1529

Grosso, N., Alves, J., Wood, K., et al. 2003, ApJ, 586, 296

Gullbring, E. 1994, A\&A, 287, 131

Gullbring, E., Petrov, P. P., Ilyin, I., et al. 1996, A\&A, 314, 835

Hamann, F., \& Persson, S. E. 1992, ApJ, 394, 628

Hamann, F. 1994, ApJS, 93, 485

Hartigan, P., Edwards, S., \& Ghandour, L. 1995, ApJ, 452, 736

Hartmann, L., Avrett, E. H., Loeser, R., \& Calvet, N. 1990, ApJ, 349, 168 
Hartmann, L., Cassen, P., \& Kenyon, S. J. 1997, ApJ, 475, 770

Henize, K. G. 1954, ApJ, 119, 459

Herbig, G. H., \& Soderblom, D. R. 1980, ApJ, 242, 628

Hirth, G. A., Mundt, R., \& Solf, J. 1997, A\&AS, 126, 437

Hughes, J., Hartigan, P., \& Clampitt, L. 1993, AJ, 105, 571

Hughes, J., Hartigan, P., Krautter, J., \& Kelemen, J. 1994, AJ, 108, 1071

Jayawardhana, R., Luhman, K. L., D’Alessio, P., \& Stauffer, J. R. 2002, ApJ, 517, L51

Johns-Krull, C. M., \& Basri, G. 1997, ApJ, 474, 433

Kaas, A. A. 1999, AJ, 118, 558

Kenyon, S. J., Brown, D. I., Tout, C. A., \& Berlind, P. 1998, AJ, 115, 2491

Kirkpatrick, J. D., Henry, T. J., \& McCarthy, D. W. 1991, ApJS, 77, 417

Kirkpatrick, J. D., Henry, T. J., \& Simons, D. A. 1995, AJ, 109, 797

Kirkpatrick, J. D., Reid, I. N., Liebert, J., et al. 1999, ApJ, 519, 802

Knude, J., \& Høg, E. 1998, A\&A, 338, 997

Königl, A. 1991, ApJ, 370, L39

Krautter, J. 1986, A\&A, 161, 195

Krautter, J. 1991, in Low Mass Star Formation in Southern Molecular Clouds, ESO Scientific Rep. No. 11

Krautter, J., Wichmann, R., Schmitt, J. H. M. M., et al. 1997, A\&AS, 123,329

Kwan, J., \& Tademaru, E. 1988, ApJ, 332, L41

Lada, C. J., \& Adams, F. C. 1992, ApJ, 393, 278

Lamzin, S. A. 1995, A\&A, 295, L20

Lamzin, S. A., Bisnovatyi-Kogan, G. S., Errico, L., et al. 1996, A\&A, 306,877

Luhman, K. L., Briceño, C., Stauffer, J. R., et al. 2003, ApJ, in press

Martín, E. L. 2000, AJ, 120, 2114

Martín, E. L., Dougados, C., Magnier, E., et al. 2001, ApJ, 561, L195

Mathis, J. S. 1990, ARA\&A, 28, 37.

McCaughrean, M. J., \& O’Dell, C. R. 1996, AJ, 111, 1977

Meyer, M. R., Calvet, N., \& Hillenbrand, L.A. 1997, AJ, 114, 288

Monin, J. L., \& Bouvier, J. 2000, A\&A, 356, L75

Murphy, D. C., Cohen, R., \& May, J. 1986, A\&A, 167, 234

Muzerolle, J., Calvet, N., \& Hartmann, L. 1998a, ApJ, 492, 743

Muzerolle, J., Hartmann, L., \& Calvet, N. 1998b, AJ, 116, 455
Muzerolle, J., Briceño, C., \& Calvet, N., et al. 2000, ApJ, 545, L141

Nakajima, Y., Tamura, M., Oasa, Y., \& Nakajima, T. 2000, AJ, 119, 873

Neuhäuser, R., Brandner, W., Alves, J., Joergens, V., \& Comerón, F. 2002, A\&A, 384, 999

Nürnberger, D., Chini, R., \& Zinnecker, H. 1997, A\&A, 324, 1036

Osterbrock, D. E. 1989, Astrophysics of gaseous nebulae and active galactic nuclei (Univ. Science Books)

Prosser, C. F., Stauffer, J. R., \& Kraft, R. P. 1991, AJ, 101, 1361

Reipurth, B., Bally, J., Graham, J. A., Lane, A. P., \& Zealey, W. J. 1986, A\&A, 164, 51

Reipurth, B., \& Zinnecker, H. 1993, A\&A, 278, 81

Rieke, G. H., \& Lebofsky, M. J. 1985, ApJ, 288, 618

Schwartz, R. D. 1977, ApJS, 35, 161

Schwartz, R. D. 1991, in Low Mass Star Formation in Southern Molecular Clouds, ESO Scientific Rep. No. 11

Shang, H., Glassgold, A. E., Shu, F. H., \& Lizano, S. 2002, ApJ, 564, 853

Siess, L., Forestini, M., \& Bertout, C. 1997, A\&A, 326, 1001

Stahler, S. W. 1983, ApJ, 274, 822.

Stapelfeldt, K. R., Krist, J. E., Ménard, F., et al. 1998, ApJ, 502, L65.

Tachihara, K., Dobashi, K., Mizuno, A., Ogawa, H., \& Fukui, Y. 1996, PASJ, 48, 489

Tachihara, K., Toyoda, S., Onishi, T., et al. 2001, PASJ, 53, 1081

Takami, M., Bailey, J., Gledhill, T. M., Chrysostomou, A., \& Hough, J. H. 2001, MNRAS, 323, 177

Takami, M., Bailey, J., \& Chrysostomou, A. 2003, A\&A, 397, 675

Thé, P.-S. 1962, Contr. Boscha Obs., 15

Whitney, B. A., Kenyon, S. J., \& Gómez M., 1997, ApJ, 485, 703

Wichmann, R., Krautter, J., Covino, E., et al. 1997, A\&A, 320, 185

Wichmann, R., Bastian, U., Krautter, J., Jankovics, I., \& Ruciński, S. M. 1998, MNRAS, 301, L39

Woitas, J., \& Leinert, Ch. 1998, A\&A, 338, 122

Wuchterl, G. 2001, in The formation of binary stars, IAU Symp. 200, ed. H. Zinnecker, \& R. D. Mathieu

Zapatero Osorio, M. R., Béjar, V. J. S., Martín, E. L., et al. 2002, ApJ, 569, L99

de Zeeuw, P. T., Hoogerwerf, R., de Bruijne, J. H. J, Brown, A. G. A., \& Blaauw, A. 1999, AJ, 117, 354 